Pacific Journal of Mathematics

A PROBLEM OF LEAST AREA 


\section{A PROBLEM OF LEAST AREA}

\section{EDWARD SilvermaN}

McShanes's solution of the Plateau problem made use of a representation theorem to reduce this problem to that of minimizing the Dirichlet integral, a non-parametric problem. The parametric integral considered by Cesari, Sigalov and Morrey $[4,16,15]$ can be interpreted as an area integral for an appropriately generalized area. If this area satisfies certain conditions there exists a Dirichlet-type integral and a representation theorem so that the McShane procedure applies. We shall restrict ourselves to such integrands since further information concerning the non-parametric problem is required to handle the general case. Results of [13] ensure the existence of a solution minimizing the Dirichlet integral and, if the integrand is sufficiently smooth, then the solution also has differentiability properties. The representation theorem is used to show that the solution which minimizes the Dirichlet integral also minimizes the parametric integral.

We use Theorem 5.2 and the representation theorem to correct an error in [21].

It seems probable that Fleming's results [9] can be combined with those of this paper to extend to surfaces of other topological types the results derived here for surfaces of the type of the disc.

It is desirable that the theory be broad enough to handle the problem of least area in $m$, the space of bounded sequences [1], hence an independent proof that the generalized area, given by the integral if the representation is good enough, is lower semi-continuous.

Since the Lebesgue area of a surface is obtained by taking the limit of the areas of a suitable sequence of approximating polyhedra, there is no loss in generality in supposing that all of the Banach spaces considered are separable, except $m$. If $B$ is such a space, then we can suppose that $B$ is a subspace of $m[17]$.

1. Let $M$ be a metric space, $C(J, M)$ be the space of continuous functions on a Jordan region $J$ into $M$ and $x \in C(J, M)$. Then there exists a monotone-light factorization $x=\lambda_{x} \mu_{x}$ such that $\mu_{x}(J) \subset m$, $L\left(\mu_{x}\right)=L(x)$, where $L$ is Lebesgue area and $\lambda_{x}$ is a contraction $[17,18]$. If $y \in C(J, M)$ then $\left\|\mu_{x}-\mu_{y}\right\| \leqq 2\|x-y\|$. If $K$ is a Jordan region contained in $J$, then $\left\|\mu_{x \mid K}(p)-\mu_{x \mid K}(q)\right\| \geqq\left\|\mu_{x}(p)-\mu_{x}(q)\right\|$ whenever $p, q \in K$. Furthermore, there exists a nonnegative valued function $\lambda$ on the subsets of $m$ such that $\lambda(A) \leqq \lambda(B)$ whenever $A \subset B$ and $\lambda\left(\mu_{x}(J)\right)=L(x)$. If $M=m$ and $x$ is monotone then $\lambda(x(J))=L(x)$

Received February 7, 1962, and in revised form July 1, 1963. 
[20]. It is not hard to verify that the last equality holds if $J$ is an admissible set [3]. In addition, if $A$ is contained in a cyclic element $C$ of $\mu_{x}(J)$ then $\lambda\left(\operatorname{Int}_{o} A\right)=\lambda(A)$ where $\operatorname{Int}_{\sigma} A$ is the interior of $A$ relative to $C$. Finally, $\lambda\left(\mu_{x}(J)\right)=\Sigma \lambda(C)$ where the summation is taken over the cyclic elements of $\mu_{x}(J)$.

A Jordan region is the homeomorph of a Jordan region in the plane. The letter $i$ will frequently be a natural number or an index but may also indicate the identity map on $m$. Thus if $\mu_{x}(J)=K$ is a Jordan region then $\mu_{x}$ and $i \mid K$ are Fréchet equivalent and $L(x)=$ $L\left(\mu_{x}\right)=\lambda(K)=L(i, K)$ where $L(i, K)=L(i \mid K)$. If a Jordan region $A$ is cut into two Jordan regions $B$ and $C$ by means of a rectifiable arc, then $\lambda(A)=\lambda(B)+\lambda(C)$.

A subset of $\mu_{x}(J)$ is open if it is open relative to $\mu_{x}(J)$. If $f$ is a function on $A$ to $B$ and $p \in B$ then $f^{\vee}(p)=\{q \in A \mid f(q)=p\}$.

Let $\mathscr{J}(x), \mathscr{X}(x), \mathscr{R}(x), \mathscr{G}(x)$ and $\mathscr{D}(x)$ be, respectively, the collection of all Jordan regions in Domain $x$, all Jordan regions in Range $\mu_{x}$, that subset of $\mathscr{P}(x)$ whose boundaries, relative to Range $\mu_{x}$, are rectifiable, the open subsets of Range $\mu_{x}$ whose boundaries are boundaries of elements of $\mathscr{R}(x)$, and the inverses, under $\mu_{x}$, of the elements of $\mathscr{G}(x)$. Thus the elements of $\mathscr{G}(x)$ and $\mathscr{D}(x)$ are open, connected and simply connected.

Now let $Q=$ Domain $x$ and $Q=\mu_{x}(Q)$, and $L(x)$ be finite.

LeMma 1.1. If $J \in \mathscr{J}(x)$ then $L\left(\mu_{x \mid J}\right)=L\left(\mu_{x}, J\right)$.

Proof. By Kolmogoroff's principle, $L(x, J) \leqq L\left(\mu_{x}, J\right) \leqq L\left(\mu_{x \mid J}\right)$. We have already noted the equality of $L(x, J)$ and $L\left(\mu_{x \mid J}\right)$.

Lemma 1.2. If $G \in \mathscr{G}(x)$ and $H=\mu_{x}^{\vee}(G)$ then $L(x, H)=\lambda(G)$.

Proof. If $J \in \mathscr{J}(x), J \subset H$, then $L(x, J)=L\left(\mu_{x \mid J}\right)=L\left(\mu_{x}, J\right)=$ $\lambda\left(\mu_{x}(J)\right) \leqq \lambda(G)$. Thus $L(x, H) \leqq \lambda(G)$ since $H$ can be invaded by Jordan regions [3]. Now let $G^{\prime}=\mathscr{Q}-G-\partial G$ and $H^{\prime}=\mu_{x}^{\vee}\left(G^{\prime}\right)$. By invading, with multiply connected Jordan regions if necessary, we obtain, as above, that $L\left(x, H^{\prime}\right) \leqq \lambda\left(G^{\prime}\right)$. Since $x \mid \partial H$ is rectifiable (thus $\lambda_{x}(\partial G)$ is also rectifiable) $L(x)=L(x, H)+L\left(x, H^{\prime}\right) \leqq \lambda(G)+\lambda\left(G^{\prime}\right)=\lambda(\mathscr{Q})=$ $L\left(\mu_{x}\right)=L(x)$ and the equality must hold throughout. The lemma follows.

LEMMA 1.3. Let $R_{1}$ and $R_{2}$ be Jordan regions with $R_{1} \subset$ Int $R_{2}$. Suppose that $x$ is light on $A=R_{2}$ - Int $R_{1}$ into $M$. Let $\mathscr{F}$ be the set of all continuous $f$ on $[0,1]$ into $A$ such that $f(0) \in \partial R_{1}$ and $f(1) \in \partial R_{2}$. Let $\mathscr{H}$ be the set of all continuous $h$ on $[0,1]$ into $A$ such that $h(0)=h(1)$ and $h(a) \neq h(b)$ unless $(a, b)=(0,1)$ or $(1,0)$, 
and $R_{1}$ is contained in the interior of the Jordan curve determined by $h$. Let $\alpha=\inf _{f \in \mathscr{F}}$ length $\mu_{x} f$ and $\beta=\inf _{h \in \mathscr{H}}$ length $\mu_{x} h$. Then $\alpha$ and $\beta$ are minima and $\beta \leqq L(x) / \alpha+\alpha$.

Proof. Let $f_{n} \in \mathscr{F}$ with length $\mu_{x} f_{n}<\alpha+1 / n$. By Hilbert's theorem concerning curves uniformly bounded in length there exists $f^{\prime}$ continuous on $[0,1]$ to which a subsequence of $\mu_{x} f_{n}$ converge in the sense of Fréchet. Since $x$ is light, $\mu_{x}^{\vee}$ is a homeomorphism. It is easy to see that $f=\mu_{x}^{\vee} f^{\prime} \in \mathscr{F}$ and length $\mu_{x} f=\alpha$.

Let $\gamma=f([0,1])$ and $q \in \operatorname{Int} A-\gamma$. It is obvious that $\gamma$ is a simple arc. There exist simple arcs $\gamma_{n}$ joining $\partial R_{1}$ to $\partial R_{2}$ and Jordan regions $J_{n}$ with $\partial J_{n} \subset \partial R_{1} \cup \partial R_{2} \cup \gamma \cup \gamma_{n}$ such that $q \in J_{n}$ and $J_{n}$ invades $A$. By [19], there exists $g_{n}$ continuous on $[0,1]$ into $J_{n}$ such that $g_{n}(0) \in \gamma$ and $g_{n}(1) \in \gamma_{n}$ such that length $\mu_{x} g_{n} \leqq L\left(x, J_{n}\right) / \alpha \leqq L(x) / \alpha$. By appealing to Hilbert's theorem again we obtain a function $g^{\prime}$ continuous on $[0,1]$ into $A$ such that $g^{\prime}(0) \in \mu_{x}(\gamma), g^{\prime}(1) \in \mu_{x}(\gamma)$ and $R_{1}$ is contained in the Jordan region $R$ bounded by $g^{\prime}([0,1])$ and the piece of $\gamma$ between $g^{\prime}(0)$ and $g^{\prime}(1)$. The lemma follows.

If $x \in C(J, m)$ then $x^{i k} \in C\left(J, E_{2}\right)$ is defined by $x^{i k}(p)=\left(x^{i}(p), x^{k}(p)\right)$ for each $p \in J$.

Lemma 1.4. Let $p \in \mathbb{Q}$ and $C$ be a cyclic element of $\mathbb{Q}$ containing $p$. Then for each $\varepsilon>0$ there exists $R \in \mathscr{R}(x)$ such that diam $R$ $<\varepsilon$ and $L(i, R)<\varepsilon$.

Proof. By [19] there exists, for each $\delta>0, R \in \mathscr{R}(x)$ with $p \in \operatorname{Int} R$ and diam $R<\delta$. By [17] there exists a number $T$ such that $L(y)>L\left(\mu_{x}\right)-\varepsilon / 2$ where $y^{j}=\left(\mu_{x}\right)^{j}$ for $j \leqq T$ and $y^{j}=0$ for $j>T$. Let $N_{i k}$ be the essential multiplicity function, [8], of $\left(\mu_{x}\right)^{i k}$. Since $\iint N_{i k}$ is finite there exists $\delta_{i k}>0$ such that $\iint_{E} N_{i k}<\varepsilon / T^{2}$ whenever $|E|<\delta_{i k}$. Let $\delta=\min \delta_{i k}, 1 \leqq i, k \leqq T$. Now take $\operatorname{diam}^{2} R<\delta / 4$. Since $\operatorname{diam}\left(\lambda_{x}\right)^{i k}(R) \leqq 2 \operatorname{diam} R, L(i, R) \leqq \sum_{1 \leqq i<j \leqq T} \iint N_{i k}+\varepsilon / 2<\varepsilon$, where the integral is taken over $\left(\lambda_{x}\right)^{i k}(R)$.

By ' $\sigma \prec \mathscr{T}^{-}(x)$ ' we mean that $\sigma$ is a finite family of non-overlapping elements of $\mathscr{T}$, where $\mathscr{T}$ is $\mathscr{J}, \mathscr{X}$, or $\mathscr{R}$; by ' $\sigma \prec \mathscr{S}(x)$ ' we mean that $\sigma$ is a finite family of pairwise disjoint elements of $\mathscr{S}$, where $\mathscr{S}$ is $\mathscr{G}$ or $\mathscr{D}$.

THEOREM 1.1. There exists $\sigma_{n} \prec \mathscr{D}(x)$ such that $\max _{D \in \sigma_{n}} \operatorname{diam} x(D) \rightarrow 0$ and $L(x)=\lim _{n} \sum_{D \in \sigma_{n}} L(x, D)$.

Proof. Let $C_{i}$ be the cyclic elements of $\mathscr{Q}$. There exists $T_{n}$ such that $\sum_{i>T_{n}} \lambda\left(C_{i}\right)<1 / n$ and $\max _{i>T_{n}} \operatorname{diam} C_{i}<1 / n$. Those points 
common to $C_{i}$ and $C_{j}, i \neq j, i, j \leqq T_{n}$, can each be placed in an element of $\mathscr{R}$ the sum of whose areas is less than $1 / n$. The part of $C_{i}, i \leqq T_{n}$, not in any of these regions can be cut up into arbitrarily small regions each of which has a rectifiable boundary, relative to $C_{i}$, by means of the intrinsic inequality. The theorem follows from the cyclic additivity of $L$ and the additivity of $L$ relative to a rectifiable cut.

If $U$ is an open subset of $Q$ let $\hat{U}=\mu_{x}^{\vee}\left(\operatorname{Int} \mu_{x}(U)\right)$ [23].

LEMMA 1.5. If $U$ is an open subset of $Q$ then $L(x, U)=\lambda\left(\mu_{x}(U)\right)$.

Proof. Let $J$ be a Jordan region (possibly multiply connected) contained in $U$. Then $\mu_{x}(J) \subset \mu_{x}(U)$ and $L(x, J) \leqq \lambda\left(\mu_{x}(U)\right)$. Hence $L(x, U) \leqq \lambda\left(\mu_{x}(U)\right)$. Let $\varepsilon>0$. The intrinsic inequality can be used to produce $\sigma \prec \mathscr{G}(x), G^{\prime}=\bigcup_{G \in \sigma} G \subset \mu_{x}(\hat{U})$, such that $\lambda\left(G^{\prime}\right)>\lambda\left(\mu_{x}(\hat{U})\right)-$ $\varepsilon$. Let $H=\mu_{x}^{\vee}\left(G^{\prime}\right)$. Then $L(x, H)=\lambda\left(G^{\prime}\right)$, by Lemma 1.2, and $H \subset U$. Thus $L(x, U) \geqq L(x, H)=\lambda\left(G^{\prime}\right)>\lambda\left(\mu_{x}(\hat{U})\right)-\varepsilon$.

LEMMA 1.6. If $U$ is an open subset of $Q$ then $L(x, \hat{U})=L(x, U)$.

Proof. $L(x, \hat{U})=\lambda\left(\mu_{x}(\hat{U})\right)=\lambda\left(\mu_{x}(U)\right)=L(x, U)$.

LEMmA 1.7. If $U \subset V \subset Q, U$ and $V$ open, then

$$
L\left(x^{i k}, V\right)-L\left(x^{i k}, U\right) \leqq L(x, V)-L(x, U) .
$$

Proof. Let $\varepsilon>0$. By Theorem 1.1 there exists $\sigma \prec \mathscr{D}(x)$, $\bigcup_{D \in \sigma} D \subset U$, and $\sum_{D \in \sigma} L(x, D)>L(x, U)-\varepsilon$. Thus

$$
\begin{aligned}
& L\left(x^{i k}, V\right)-L\left(x^{i k}, U\right) \leqq L\left(x^{i k}, V\right)-\sum_{D \in \sigma} L\left(x^{i k}, D\right) \\
& \quad=L\left(x^{i k}, V-\bigcup_{D \in \sigma} D\right) \leqq L\left(x, V-\bigcup_{D \in \sigma} D\right) \\
& \quad \leqq L(x, V)-\sum_{D \in \sigma} L(x, D)<L(x, V)-L(x, U)+\varepsilon .
\end{aligned}
$$

2. If $B$ is a Banach space over the reals, then $B_{1}$ is the set of all elements of $B$ having norm one and $B^{*}$ is the space of continuous linear functionals over $B$. For the purposes of this paper there is no loss in generality in supposing that $B$ is separable. In this case there exist $f_{n} \in B_{1}^{*}, n=1,2, \cdots$, such that $\|a\|=\sup _{n}\left[a, f_{n}\right]$. By identifying $a \in B$ with $\left\{\left[a, f_{n}\right]\right\} \in m$ we can suppose that $B$ is a subspace of $m$ [17]. Let $m^{\prime}$ be the space of bounded functions $\beta$ on $N \times N, N$ is the set of positive integers, such that $\beta(m, n)+\beta(n, m)=0$. If $a, b \in m$ then we define $a \wedge b \in m^{\prime}$ by $a \wedge b(m, n)=a^{m} b^{n}-a^{n} b^{m}$, where $a=\left\{a^{i}\right\}$ and $b=\left\{b^{i}\right\}$. Thus we suppose that the exterior product of $B$ with 
itself, $B \wedge B$, is contained in $m^{\prime}$. If $\beta \in m^{\prime}$ then we put $\|\beta\|=$ $\sup |\beta(m, n)|$ and obtain $\|a \wedge b\| \leqq 2\|a\|\|b\| \leqq\|a\|^{2}+\|b\|^{2}$. If $a \wedge b=0$ then $a$ and $b$ are linearly dependent, and conversely. We will usually write $\beta^{i k}$ for $\beta(i, k)$.

If $f$ is continuous on a Jordan region $J$ into the plane then $O(f, \partial J, p)$ is the topological index of $p$ relative to $f(\partial J)$.

LEMMA 2.1. If $U$ is an open connected subset of $Q$ and if $J_{n}$ is a sequence of (possibly multiply-connected) Jordan regions invading $U$ then $\lim \iint O\left(x^{i k}, \partial J_{n}\right)$ exists. The limit is independent of the sequence $\left\{J_{n}\right\}$.

Proof. Let $\varepsilon>0$ and take $K$ so large that $L\left(x, J_{r}\right)>L(x, U)-\varepsilon / 2$ for $r>K$. If $n>m>K$ then

$$
\begin{aligned}
& \left|\iint O\left(x^{i k}, \partial J_{n}\right)-\iint O\left(x^{i k}, \partial J_{m}\right)\right|-\varepsilon / 2<\left|\iint O\left(x^{i k}, \partial\left(J_{n}-\operatorname{Int} J_{m}\right)\right)\right| \\
& \quad \leqq \iint\left|O\left(x^{i k}, \partial\left(J_{n}-\operatorname{Int} J_{m}\right)\right)\right| \leqq L\left(x^{i k}, J_{n}-\operatorname{Int} J_{m}\right) \\
& \quad \leqq L\left(x, J_{n}-\operatorname{Int} J_{m}\right) \leqq L\left(x, J_{n}\right)-L\left(x, J_{m}\right)<\varepsilon .
\end{aligned}
$$

Let $\alpha_{n}^{i k}=\iint O\left(x^{i k}, \partial J_{n}\right)$. Then $\left\{\alpha_{n}^{i k}\right\}$ is a Cauchy sequence for each $(i, k)$. Let $\alpha^{i k}=\lim \alpha_{n}^{i k}$. Clearly $\alpha_{n} \in m^{\prime}, \alpha \in m^{\prime}$, and $\alpha_{n} \rightarrow \alpha$ in $m^{\prime}$. The last statement is evident.

If $U$ is an open connected subset of $Q$ then we define $\langle x, U\rangle=$ $\alpha$. We may write $\langle x, J\rangle$ for $\langle x$, Int $J\rangle$ when $J$ is a Jordan region. Thus we have just shown that $\|\langle x, U\rangle-\langle x, J\rangle\| \leqq L(x, U)-L(x, J)$ if $J \subset U$. If $U$ is not connected we put $\langle x, U\rangle=\Sigma\langle x, W\rangle$ where the sum is taken over the components $W$ of $U$.

LEMMA 2.2. If $U \subset V$ are open subsets of $Q$ then

$$
\|\langle x, V\rangle-\langle x, U\rangle\| \leqq L(x, V)-L(x, U) \text {. }
$$

Lemma 2.3. If $U$ is an open subset of $Q$ and if $D \subset U, D \in \mathscr{D}(x)$, then $\langle x, U\rangle=\langle x, D\rangle+\langle x, U-\operatorname{Clos} D\rangle$.

The proof depends upon the fact that the image of $\partial D$ under $x$ is rectifiable.

The monotone map $\mu_{x}$ induces an orientation of the cyclic elements of $\mathscr{Q}$ [24]. We assume from now on that $\mathscr{Q}$ is a subset of $m$ together with this induced orientation.

LEMMA 2.4. Let $r_{\sigma}$ be the monotone retraction of $\mathbb{Q}$ onto one of its cyclic elements $C$. If $J$ is a Jordan region contained in $Q$ then 
$O\left(x^{i k}, \partial J\right)=\Sigma O\left(\left(\lambda_{x}\right)^{i k} r_{c}, \mu_{x}(\partial J)\right)$, where the sum is taken over the cyclic elements of $\mathbb{Q}$.

Proof. Let $\delta \subset \mu_{x}(\partial J)$ and suppose that $r_{o}$ is constant on $\delta$ and that $r_{\sigma}=i$ on $\mu_{x}(\partial J)-\delta$. Let $E$ be the oriented plane containing $x^{i k}(Q), P \in E$, and $L$ be a half-line in $E$ terminating at $P$. If $p \in \partial J$ and $f$ is continuous on $J$ into $E$, let $w(f, p)$ be the angle between the half-line determined by $f(p)$ and $P$ with $L$. Evidently the change in $w\left(x^{i k}\right)$ around $\partial J$ is equal to the sum of the changes of $w\left(\left(\lambda_{x}\right)^{i k} r_{o}\right)$ on $\mu_{x}(\partial J)$. Thus $\langle x, J\rangle=\Sigma\left\langle\lambda_{x} r_{o}, \mu_{x}(J)\right\rangle$.

LEMMA 2.5. $\langle x, U\rangle=\left\langle\lambda_{x}, \mu_{x}(\hat{U})\right\rangle$.

Proof. We can suppose that $U$ is connected. Let $\varepsilon>0$. There exists a Jordan region $J \subset U$ with $L(x, U)<L(x, J)+\varepsilon$. If $K=\operatorname{Int} J$ then

$$
\begin{aligned}
& \sum_{\sigma}\left[L\left(\lambda_{x} r_{o}, \mu_{x}(\hat{U})\right)-L\left(\lambda_{x} r_{o}, \mu_{x}(\hat{K})\right)\right] \\
& \quad=L\left(\lambda_{x}, \mu_{x}(\hat{U})\right)-L\left(\lambda_{x}, \mu_{x}(\hat{K})\right)=L(x, U)-L(x, J)<\varepsilon .
\end{aligned}
$$

Hence

$$
\begin{aligned}
& \left\|\langle x, U\rangle-\left\langle\lambda_{x}, \mu_{x}(\hat{U})\right\rangle\right\| \leqq\|\langle x, U\rangle-\langle x, J\rangle\| \\
& \quad+\left\|\langle x, J\rangle-\Sigma\left\langle\lambda_{x} r_{o}, \mu_{x}(J)\right\rangle\right\|+\left\|\Sigma\left\langle\lambda_{x} r_{\theta}, \mu_{x}(\hat{U})\right\rangle-\Sigma\left\langle\lambda_{x} r_{o}, \mu_{x}(J)\right\rangle\right\| \\
& \quad+\left\|\Sigma\left\langle\lambda_{x} r_{\theta}, \mu_{s}(U)\right\rangle-\left\langle\lambda_{x}, \mu_{x}(\hat{U})\right\rangle\right\|\langle\varepsilon+0+\varepsilon+0=2 \varepsilon .
\end{aligned}
$$

LEMma 2.6. Let $\mu_{x}(U)$ contain a cyclic element $C$ of the type of a sphere. Then $\left\langle\lambda_{x} r_{o}, \mu_{x}(U)\right\rangle=0$.

Proof. Since $U$ is open and $\mu_{x}^{\vee}(C) \subset U$ there exists a Jordan region $J$ with $\mu_{x}^{\vee}(C) \subset J \subset U$. Hence, since $r_{\sigma}$ is the identity on $C$, $\left\langle\lambda_{x} r_{c}, \mu_{x}(J)\right\rangle=\left\langle\lambda_{x}, r_{o} \mu_{x}(J)\right\rangle=0$.

LEMMA 2.7. If $x$ is constant on $\partial Q$ then $\langle x, Q\rangle=0$, furthermore $\langle x, Q\rangle=\left\langle\lambda_{x} r_{O_{0}}, \mu_{x}(Q)\right\rangle$ if $C_{0}$ is the cyclic element containing $\mu_{x}(\partial Q)$. Finally, $\langle x, U\rangle=\Sigma\left\langle\lambda_{x} r_{0}, \mu_{x}(U)\right\rangle$ where the sum extends only over those cyclic elements of $Q^{3}$ not contained in $\mu_{x}(U)$ plus, possibly, $C_{0}$.

Lemma 2.8. Let $C$ and $C^{\prime}$ be two Fréchet curves in $E_{2}$, of the type of the circle, each of whose lengths is less than $M$. Then

$$
\left|\iint_{E_{2}}\left[O(C, p)-O\left(C^{\prime}, p\right)\right] d p\right| \leqq 2 M\left\|C, C^{\prime}\right\|
$$

where $O(C, p)$ is the topological index of $p$ relative to $C$ and $\left\|C, C^{\prime}\right\|$ 
is the Fréchet distance between $C$ and $C^{\prime}$.

Proof. Choose $\varepsilon>0$. There exist functions $f, g, f^{\prime}$ and $g^{\prime}$ on $[0,1]$, all continuous and of bounded variation such that $\left\|f-f^{\prime}\right\|+$ $\left\|g-g^{\prime}\right\|<\left\|C, C^{\prime}\right\|+\varepsilon$ and

$$
\begin{aligned}
& \left|\iint\left[O(C, p)-O\left(C^{\prime}, p\right)\right] d p\right|=\left|\int_{0}^{1} f d g-\int_{0}^{1} f^{\prime} d g^{\prime}\right| \\
& \quad=\left|\int_{0}^{1}\left(f-f^{\prime}\right) d g+\int_{0}^{1}\left(g-g^{\prime}\right) d f^{\prime}\right|<2 M\left\{\left\|C, C^{\prime}\right\|+\varepsilon\right\} .
\end{aligned}
$$

We require an additional property of $\mu_{x}$. If $p, q \in Q$ then $\left\|\mu_{x}(p)-\mu_{x}(q)\right\|=\inf \{$ length $x g \mid g$ is continuous on $[0,1]$ into $Q$ with $g(0)=p$ and $g(1)=q\}$.

THEOREM 2.1. Suppose that $x_{n} \rightarrow x$ in $C(Q, m)$ and that there exists a number $M$ such that $L\left(x_{n}\right)<M$ for all $n$. Then for each simply connected Jordan region $J_{0} \subset Q$ there exists a finite collection $\sigma_{n}$ of non-overlapping simply-connected Jordan regions in $J_{0}$ such that

$$
\lim \sum_{J \in \sigma_{n}}\left\langle x_{n}, J\right\rangle=\left\langle x, J_{0}\right\rangle \text {. }
$$

Proof. If the theorem were false there would exist $\varepsilon_{0}>0$ such that limsup $\left.\left|\sum_{J \in \sigma_{n}}\left\langle x_{n}, J\right\rangle-\left\langle x, J_{0}\right\rangle\right|\right\rangle \varepsilon_{0}$ for each admissible $\left\{\sigma_{n}\right\}$. By extracting a suitable subsequence we can suppose that the limit exists.

Let $C_{k}$ be the cyclic elements of $\mathscr{Q}$ and $r_{k}$ be the monotone retraction of $\mathscr{Q}$ onto $C_{k}$. There exists a number $T$ such that $\sum_{k>T} \lambda\left(C_{k}\right)<\varepsilon_{0} / 4$. For each $k \leqq T$ let $K_{j}(k)$ be the Jordan regions in $C_{k}$ whose boundaries, relative to $C_{k}$ are subsets of $r_{k} \mu_{x}\left(\partial J_{0}\right)$. There exists a number $s_{k}$ such that $\sum_{j>s_{k}} \lambda\left(K_{j}(k)\right)<\varepsilon_{0} /(3 T)$. Let $s=\max s_{k}$ and $\eta=\varepsilon_{0} /(4 T s)$.

Let us fix $k \leqq T, j \leqq s_{k}$ and write $K$ for $K_{j}(k)$. There exist Jordan regions $K_{i} \subset K, i=1,2,3$, with $K_{1} \subset K_{2}-\partial K_{2} \subset K_{2} \subset K_{3}-\partial K_{3}$ $\subset K_{3} \subset K-\partial K$, where boundaries are taken relative to $C_{k}$, such that $\lambda\left(K_{1}\right)>\lambda(K)-\eta$. Let $\psi=\inf \operatorname{diam} A$ where $A$ is a continuum in $K_{3}-K_{2}$ which separates $\partial K_{3}$ from $\partial K_{2}$. Evidently $\psi>0$. Let $\xi(j, k)=(1 / 6) \min \left\{1, \operatorname{dist}\left(\partial K_{1}, \partial K_{2}\right)\right.$, dist $\left(\partial K_{2}, \partial K_{3}\right)$, dist $\left.\left(\partial K_{3}, \partial K\right), \psi\right\}, \xi=$ $\min \xi(j, k), N=M / \xi+1$, and $\zeta=\min \{\xi, \eta /(4 N)\}$. We consider only such $n$ for which $\left\|x_{n}-x\right\|<\zeta$.

Let $T_{i}=\left(r_{k} \mu_{x}\right)^{2}\left(K_{i}-\partial K_{i}\right)$. Then $T_{i}$ is an open two-cell and there exist Jordan regions $S_{2}$ and $S_{3}$ such that $T_{2} \subset S_{2} \subset S_{3} \subset T_{3}$ and $\operatorname{dist}\left(\mu_{x}\left(\partial S_{2}\right), \mu_{x}\left(\partial S_{3}\right)\right)>5 \xi$. Let $B_{n}^{i}=\mu_{x_{n}}\left(\partial S_{i}\right)$. Then $\operatorname{dist~}\left(B_{n}^{2}, B_{n}^{3}\right)>$ $5 \xi-4 \zeta \geqq \xi$.

If $p$ is a cutpoint of $\mathscr{Q}_{n}=\mu_{x_{n}}(Q)$ on $B_{n}^{3}$ and if $C^{\prime}(n)$ and $C^{\prime \prime}(n)$ are two cyclic elements of $\mathscr{Q}_{n}$ separated by $p$ then only one is not 
separated from $B_{n}^{2}$ by $p$. Hence there is a single cyclic element $C(n)$ of $Q_{n}$ such that no cut point of $\mathbb{Q}_{n}$ on $B_{n}^{3}$ separates $C(n)$ from $B_{n}^{2}$. Let $r(n)$ be the monotone retraction of $\mathscr{Q}_{n}$ onto $C(n)$.

If $B_{n}^{2} \cap C(n)=0$ there is a cutpoint $p$ between $C(n)$ and $B_{n}^{2}$. Hence $p \notin B_{n}^{3}$ and, consequently, $p$ separates $B_{n}^{2}$ from $B_{n}^{3}$. This implies that there exists a continuum $A \subset K_{3}-K_{2}$ which separates $\partial K_{3}$ from $\partial K_{2}$ and whose diameter is less than $4 \xi$, but this is a contradiction. Hence $B_{n}^{2} \cap C(n) \neq 0$ and, therefore, $\operatorname{dist}\left(r(n) B_{n}^{3}, r(n) B_{n}^{2}\right) \geqq \operatorname{dist}\left(B_{n}^{3}, B_{n}^{2}\right)>\xi$. Thus there exist Jordan regions $R_{n}^{2} \subset R_{n}^{3} \subset C(n)$ such that $r(n) B_{n}^{2} \subset R_{n}^{2}$, $r(n) B_{n}^{3} \cap R_{n}^{3}=0$ and dist $\left(\partial R_{n}^{2}, \partial R_{n}^{3}\right)>\xi$. Since $\lambda\left(R_{n}^{3}\right)<\lambda(C(n))<M$ there exists a Jordan region $R(n)$ with $R_{n}^{2} \subset R(n) \subset R_{n}^{3}$ and length $\partial R(n)<N$.

The curves $\{\partial R(n)\}$ lie in a compact set and are uniformly bounded in length by $N$; thus there exist representations $\gamma_{n} \in R(n)$ on $[0,1]$ such that $\gamma_{n}$ converges uniformly to a continuous function $\gamma$ on $[0,1]$ onto $Q$. From now on suppose that $n$ is so big that $\left\|\gamma_{n}-\gamma\right\|<\zeta$. Let $t \in[0,1], p=\gamma(t)$ and $p_{n}=\gamma_{n}(t)$. There exists $q_{n} \in S_{3}-S_{2}$ such that $p_{n}=\mu_{x_{n}}\left(q_{n}\right)$. Thus $\left\|p-\mu_{x}\left(q_{n}\right)\right\| \leqq\left\|p-p_{n}\right\|+\left\|\mu_{x_{n}}\left(q_{n}\right)-\mu_{x}\left(q_{n}\right)\right\| \leqq$ $\zeta+2 \zeta=3 \zeta$. First suppose that $\mu_{x}\left(q_{n}\right) \in C_{k}$. Then $\mu_{x}\left(q_{n}\right) \in K_{3}-K_{2}$. Whether $p \in C_{k}$ or not, $\left\|r_{k}(p)-\mu_{x}\left(q_{n}\right)\right\| \leqq\left\|p-\mu_{x}\left(q_{n}\right)\right\|<3 \zeta$. Now suppose that $\mu_{x}\left(q_{n}\right) \notin C_{k}$. If $r_{k} \mu_{x}\left(q_{n}\right)$ separates $p$ from $C_{k}$ then $r_{k} \mu_{x}\left(q_{n}\right)=$ $r_{k}(p)$; otherwise $\left\|r_{k}(p)-r_{k} \mu_{x}\left(q_{n}\right)\right\|<\left\|p-\mu_{x}\left(q_{n}\right)\right\|<3 \zeta$. Thus if $p=$ $\gamma(t)$ and $q \in q(t)=r_{k} \mu_{x} \mu_{x_{n}}^{v} \gamma_{n}(t)$ then $\|p-q\|<3 \zeta$. Let $A(n)=\cup q(t)$. Then $A(n) \subset K_{3}-K_{2}, \gamma([0,1]) \subset K-K_{1}$, and $A(n)$ separates $\partial K_{3}$ from $\partial K_{2}$. If $r_{k} \gamma$ could be shrunk to a point in $K_{3}-K_{2}$ then $\gamma$ could be in $Q^{-}-K_{2}$. Now $B_{n}=\bigcup_{t} \mu_{x_{n}} \mu_{x}^{v} \gamma(t)$ would be shrunk to a set of arbitrary small diameter in $C(n)-R_{n}^{2}$, for $n$ large enough, and this is impossible since $\operatorname{diam} R_{n}^{2} \geqq \operatorname{diam} K_{2}-2\left\|x-x_{n}\right\|$.

Let $G_{j}$ be the components of $K-\gamma([0,1])$ which do not contain $\partial K$. Then

$$
\iint O\left(\pi^{i k} \gamma,[0,1]\right)=\iint O\left(\pi^{i k}, \gamma([0,1])\right)=\Sigma\left\langle\lambda_{x}, G_{j}\right\rangle^{i k}
$$

and

$$
\iint O\left(\pi^{i k} \gamma_{n},[0,1]\right)=\left\langle\lambda_{x_{n}}, R(n)\right\rangle^{i k},
$$

where $\pi^{i k}(\alpha)=\left(a^{i}, a^{k}\right)$. By Lemma 2.8,

$$
\left|\iint O\left(\pi^{i k} \gamma_{n},[0,1]\right)-O\left(\pi^{i k} \gamma,[0,1]\right)\right|<4 N \zeta .
$$

Let the subscripts of the $G$ 's be so chosen that $G_{1} \supset K_{1}$. Then

$$
\left\|\left\langle\lambda_{x}, K\right\rangle-\left\langle\lambda_{x}, G_{1}\right\rangle\right\|<\lambda(K)-\lambda\left(G_{1}\right) \leqq \lambda(K)-\lambda\left(K_{1}\right)<\eta .
$$


Hence $\left\|\left\langle\lambda_{x_{n}}, R(n)\right\rangle-\left\langle\lambda_{x}, K\right\rangle\right\|\langle 4 N \zeta+\eta\langle 2 \eta$.

Next, $\left\langle\lambda_{x_{n}}, R(n)\right\rangle=\left\langle\lambda_{x_{n}}, r(n)^{\vee} R(n)\right\rangle=\left\langle x_{n}, D_{n}\right\rangle \quad$ where $\quad D_{n}=$ $\mu_{x_{n}}^{\vee}\left[\operatorname{Int} r(n)^{\vee} R(n)\right]$. There exists a simply connected Jordan region $J_{n} \subset D_{n}$ such that $\left\|\left\langle x_{n}, D_{n}\right\rangle-\left\langle x_{n}, J_{n}\right\rangle\right\|\left\langle\eta\right.$. Thus $\left\|\left\langle\lambda_{x}, K\right\rangle-\left\langle x_{n}, J_{n}\right\rangle\right\|\langle 3 \eta$.

Let $r^{\prime}(n), R^{\prime}(n), J^{\prime}(n), K^{\prime}$ and $K_{3}^{\prime}$ correspond to $K_{j^{\prime}}\left(k^{\prime}\right)$ as $r(n)$, $R(n), J(n), K$ and $K_{3}$ correspond to $K_{j}(k)$ and suppose either $k \neq k^{\prime}$ or $j \neq j^{\prime}$. If $R(n) \cap R^{\prime}(n) \neq 0$ there exists $p \in R(n) \cap R^{\prime}(n), q \in K_{3}$ and $q^{\prime} \in K_{3}^{\prime}$ such that $\|p-q\|<2 \zeta_{n}$ and $\left\|p-q^{\prime}\right\|<2 \zeta_{n}$. This gives $4 \zeta \geqq 4 \zeta_{n}>\left\|q-q^{\prime}\right\|>\operatorname{dist}\{q, \partial K\} \geqq \operatorname{dist}\left\{\partial K_{3}, \partial K\right\}>5 \xi>4 \zeta$. Hence $R(n) \cap R^{\prime}(n)=0, r(n) R(n) \cap r^{\prime}(n) R^{\prime}(n)=0$ and, finally, $J(n) \cap J^{\prime}(n)=0$.

Now let $\sigma_{n}$ be the collection of all such $J_{n}$, one for each $K_{j}(k)$. By some arithmetic we get

$$
\left\|\sum_{J \in \sigma_{n}}\left\langle x_{n}, J\right\rangle-\left\langle x, J_{0}\right\rangle\right\|\left\langle\varepsilon_{0}\right. \text {. }
$$

COROLlaRY. If $x_{n} \rightarrow x, L\left(x_{n}\right)<M$ and $\sigma \prec \mathscr{J}(x)$ then there exists $\sigma_{n} \prec \mathscr{J}\left(x_{n}\right)=\mathscr{J}(x)$ such that $\lim \sum_{J \in \sigma_{n}}\left\langle x_{n}, J\right\rangle=\sum_{J \in \sigma}\langle x, J\rangle$.

3. If $a \in m$ let $\pi_{n}(a)={ }_{n} a=\left\{{ }_{n} a^{i}\right\}$ where ${ }_{n} a^{i}=a^{i}$ or 0 according as $i \leqq n$ or $i>n$. If $\alpha \in m^{\prime}$ let ${ }_{n} \alpha^{i k}=\alpha^{i k}$ or 0 according as $i, k \leqq n$ or either $i$ or $k>n$. Let ${ }_{n} m=\pi_{n}(m)$ and ${ }_{n} m^{\prime}={ }_{n} m \wedge{ }_{n} m$. We recall that we can suppose that ${ }_{n} m^{\prime} \subset m^{\prime}$.

Let $\psi$ be a nonnegative valued function on ${ }_{n} m^{\prime}$, for some natural number $n$, with the following properties:

(i) $\psi$ is continuous,

(ii) $\psi$ is positively homogeneous of degree one,

(iii) $\psi$ is convex, and

(iv) there exist $K \geqq k>0$ such that $k\|\alpha\| \leqq \psi(\alpha) \leqq K\|\alpha\|$ for all $\alpha \in{ }_{n} m^{\prime}$. Let $F_{n}^{\prime}$ be the collection of such functions $\psi$.

Let $\mathscr{A}^{\prime}$ be the set of all $\psi$ defined on $m^{\prime}$ with the property that $\left.\psi_{n}=\psi \mid{ }_{n} m^{\prime}\right) \in F_{n}^{\prime}$ and $\psi_{n} \leqq \psi_{n+1}, \psi_{n} \rightarrow \psi$.

Let $\psi \in \mathscr{A}^{\prime}$. If there exist $S \subset m^{*} \times m^{*}$ such that $\psi(a \wedge b)=$ $\sup \{[a \wedge b, f \wedge g] \mid(f, g) \in S\}$, where $[a \wedge b, f \wedge g]=f(a) g(b)-f(b) g(a)$, then $\psi$ is simple. If $\psi(a \wedge b)=[a \wedge b, f \wedge g]$ for some $(f, g) \in S$ then we write $(f, g) \in S(a \wedge b)$.

We now norm $m \times m^{\prime}$ and $m \times m^{\prime *}$ by $\|(a, \alpha)\|=\|a\|+\|\alpha\|$ and $\|(a, \zeta)\|=\|a\|+\|\zeta\|$ where $a \in m, \alpha \in m^{\prime}, \zeta \in m^{\prime *}$ and $\|\quad\|$ is the appropriate norm.

Suppose that $A$ is a bounded closed subset of ${ }_{n} m$ for some $n$ and $\psi$ is a real-valued continuous function on $A \times{ }_{n} m^{\prime}$ with $\psi_{n} \in F_{n}^{\prime}$, where $\psi_{n}(\alpha)=\psi(\alpha, \alpha)$ for each $a \in A$ and $\alpha \in_{n} m^{\prime}$, with $k$ and $K$ of (iv) independent of $a$. 
LEMma 3.1. Let $\psi$ be as in the last paragraph. Then $\psi$ has an extension $\psi_{0}$ on ${ }_{n} m \times{ }_{n} m^{\prime}$ which satisfies all of the conditions imposed on $\psi$ with $A$ replaced by ${ }_{n} m$.

Proof. Let $h$ be defined on $A \times{ }_{n} m_{1}^{* *}$ by

$$
\frac{1}{h(\alpha, \zeta)}=\max _{0 \neq \alpha \in \in_{n} m^{\prime}} \frac{[\alpha, \zeta]}{\psi(\alpha, \alpha)} \text {. }
$$

It is easy to see that $k \leqq h(a, \zeta) \leqq K$ and that $h$ is uniformly continuous. The McShane Extension Theorem (15], applied to $h$, yields an extension $k$ defined on ${ }_{n} m \times{ }_{n} m_{1}^{\prime *}$ which has the same bounds and modulus of continuity as $h$. Let $\psi_{0}(\alpha, \alpha)=\max \left\{k(\alpha, \zeta)[\alpha, \zeta] \mid \zeta \in m_{1}^{\prime *}\right\}$.

Let $F_{n}$ be the collection of all such functions $\psi_{0}$. Let $\mathscr{A}$ be the set of all $\psi$ defined on $m \times m^{\prime}$ such that $\psi_{a} \in \mathscr{A}^{\prime}$, there exists $K \geqq$ $k>0$ with $k\|\alpha\| \leqq \psi(\alpha, \alpha) \leqq K\|\alpha\|$ for all $a \in m, \alpha \in m^{\prime}, \quad \psi_{n}=$ $\psi \mid\left({ }_{n} m \times{ }_{n} m^{\prime}\right) \in F_{n}$ and $\psi_{n} \leqq \psi_{n+1}, \psi_{n} \rightarrow \psi$.

Let $\psi \in \mathscr{A}, \alpha_{p}$ and $\alpha$ in $m^{\prime}$ and suppose that $\alpha_{p}^{i k} \rightarrow \alpha^{i k}$ for all $(i, k)$. Then ${ }_{n} \alpha_{p} \rightarrow{ }_{n} \alpha$ for all $n$. Hence $\psi_{n}\left({ }_{n} \alpha,{ }_{n} \alpha\right)=\lim \psi_{n}\left({ }_{n} \alpha,{ }_{n} \alpha_{p}\right) \leqq$ $\liminf \psi\left(\alpha, \alpha_{p}\right)$. Thus $\psi(\alpha, \alpha) \leqq \liminf \psi\left(\alpha, \alpha_{p}\right)$.

Suppose that $\psi_{n} \in F_{n}$ with $k\left\|_{n} \alpha\right\| \leqq \psi_{n}\left(_{n} a,{ }_{n} \alpha\right) \leqq K\left\|{ }_{n} \alpha\right\|$ for all $(\alpha, \alpha)$. Let $\psi_{p}\left({ }_{p} a,{ }_{p} \alpha\right)=\max \left(\psi_{n}\left({ }_{n} a,{ }_{n} \alpha\right), k\left\|_{p} \alpha\right\|\right)$ for $p>n$. Then $\psi_{p} \in F_{p}$ for such $p$ and $\psi=\lim \psi_{p}$ is in $\mathscr{A}$. Furthermore, $\psi$ is an extension of $\psi_{n}$ and is simple if $\psi_{n}$ is.

If $x \in C(Q, m)$ we define, for each $\psi \in \mathscr{A}$,

$$
P(\psi, x)=\lim _{\|\sigma\| \rightarrow 0} \sum_{J \in \sigma} \min _{p \in J} \psi(x(p),\langle x, J\rangle), \quad \sigma \prec \mathcal{J}(x) .
$$

Suppose that $\psi \in \mathscr{A}$. It is easy to verify that $P(\psi, x)=$ $\lim P\left(\psi_{n}, x\right)$.

In [17] the definition for the Peano area of $x$ was equivalent to

$$
P(x)=\sup _{\sigma<\mathcal{J}(x)} \sum_{J \in \sigma} \sup _{i k} \iint\left|0\left(x^{i k}, \partial J\right)\right| .
$$

If Range $x$ is contained in a Euclidean space (which we can suppose is contained in $m$ ) then $P(x)$ is the usual Peano area of $x$. Let $\psi_{0}(\alpha, \alpha)=\|\alpha\|$. Evidently $\psi_{0} \in \mathscr{A}$.

LeMma 3.2. $P\left(\psi_{0}, x\right)=P(x)$.

An inequality in one direction is obvious. The difficult inequality follows from a result of Cesari $[U=V, 3]$.

LEMMA 3.3. $\quad P(\psi, x)=\lim _{\|\sigma\| \rightarrow 0} \sum_{T \in \sigma} \inf _{\mu_{x}(p) \in T} \psi\left(x(p),\left\langle\lambda_{x}, T\right\rangle\right)$ where $\sigma \prec \mathscr{K}(x), \mathscr{R}(x)$, or $\mathscr{G}(x)$, and 


$$
P(\psi, x)=\lim _{\|\sigma\| \rightarrow 0} \sum_{D \in \alpha} \inf _{p \in D} \psi(x(p),\langle x, D\rangle), \quad \sigma \prec \mathscr{D}(x) .
$$

The proof makes use only of the definitions and the results connecting $\langle x, J\rangle$ with $\left\langle\lambda_{x}, \mu_{x}(J)\right\rangle$.

Lemma 3.4. If $x$ and $y$ are Fréchet equivalent then $P(\psi, x)=$ $P(\psi, y)$. Furthermore, $P(\psi, \cdot)$ is cyclically additive.

We understand that the space of Fréchet surfaces is given the Fréchet metric.

If $\mathscr{S}$ is a Fréchet surface then $P(\psi, \mathscr{S})=P(\psi, x)$ for any $x \in \mathscr{S}$.

THEOREM 3.1. $P(\psi, \cdot)$ is lower semi-continuous both on $C(Q, m)$ and on the space of Fréchet surfaces.

The results of the Corollary to Theorem 2.1 enable us to use the standard arguments.

THEOREM 3.2. If $x_{n}^{i}$ converges uniformly to $x^{i}$ for each $i$, then $P(\psi, x) \leqq \liminf P\left(\psi, x_{n}\right)$.

There is nothing to show if $\psi \in F_{n}$ for some $n$. Otherwise we make use of the fact that the limit of an increasing sequence of lower semi-continuous functions is again lower semi-continuous.

4. Our next step is to show that the expected formula holds for $P(\psi, x)$ whenever $x$ is smooth enough. What follows is drawn from [4; $\S \S 28,30,32,12.10$ and 12.12].

Let $x \in C(Q, m)$. If $L\left(x^{i k}\right)<\infty$ then the interval function whose value on $R$ is $\langle x, R\rangle^{i k}$ is differentiable and its derivative $\mathscr{J}\left(x^{i k}\right)$ is the generalized Jacobian of $x^{i k}=\left(x^{i}, x^{k}\right)$. If $x^{i}$ and $x^{k}$ have ordinary first derivatives almost everwhere in Int $Q$ then $\mathscr{J}\left(x^{i k}\right)=x_{u}^{i} x_{v}^{k}-x_{v}^{i} x_{u}^{k}$ almost everwhere. We define the generalized Jacobian of $x$ on $m^{\prime}$ by $[\mathscr{J}(x)]^{i k}=\mathscr{J}\left(x^{i k}\right)$. Since $P(\psi, x)$ is superadditive, it has a derivative almost everywhere. By Jensen's inequality,

$$
\iint_{q} \psi(x, \mathscr{J}(x)) \geqq \min _{r \in q} \psi\left(x(r), \iint_{q} \mathscr{J}(x)\right)
$$

for each square $q$ contained in Int $Q$. It follows that $P^{\prime}(\psi, x, p) \geqq$ $\psi(x(p), \mathcal{J}(x, p))$ almost everywhere. Thus $P(\psi, x) \geqq \iint \psi(x, \mathscr{J}(x))$. We wish to show that the equality holds for $x$ sufficiently smooth. The proper requirement would be absolute continuity in the sense of Cesari, but we shall content ourselves with showing that the equality holds if $x$ is a $D$-map [21]. 
THEOREM 4.1. If $x$ is a $D$-map on a Jordan region $Q$ and $\psi \in \mathscr{A}$ then $P(\psi, x)=\iint_{\operatorname{Int} Q} \psi(x, \mathscr{J}(x))$.

Proof. Since $x$ is a $D$-map, $P(\psi, x \mid \cdot)$ is an absolutely continuous interval function. Hence, by the preceding paragraph, we need only show that $P(\psi, x) \leqq \iint \psi(x, \mathcal{J}(x))$. Furthermore, if $\varepsilon>0$, then there exists a finite collection $\sigma$ of non-overlapping squares contained in Int $Q$ such that

$$
\begin{aligned}
& P(\psi, x)-\varepsilon<\sum_{q \in \sigma} \min _{p \in q} \psi\left(x(p), \iint_{q} \mathcal{J}(x)\right) \\
& \quad \leqq \sum_{q \in \sigma} \iint_{q} \psi(x, \mathcal{J}(x)) \leqq \iint_{\mathrm{Int} Q} \psi(x, \mathscr{J}(x))
\end{aligned}
$$

where we have used the fact that $x$ is a $D$-map to obtain $\sigma$ and the equality of $\langle x, q\rangle$ and $\iint_{q} \mathscr{J}(x)$.

If $\mathscr{P}$ is a polyhedron then there exists a quasilinear representation $x$ of $\mathscr{P}$ on the unit square and $P(\psi, \mathscr{P})=\iint \psi(x, \mathscr{J}(x))$. Since $\iint\|\mathscr{J}(x)\|$ is often referred to as the elementary area of $\mathscr{P}$, we shall regard $\iint \psi(x, \mathscr{J}(x))$ as the elementary area of $\mathscr{P}$ corresponding to the (nongeometric) area $\psi$. Let us write $\mathscr{E}(\psi, \mathscr{P})$ for this quantity. That the elementary area is lower semi-continuous with respect to Fréchet convergence follows from Theorems 3.1 and 4.1.

Now let $\mathscr{S}$ be a Fréchet surface of the type of the two-cell. We define a Lebesgue area for $\mathscr{S}$ as follows:

$$
L(\psi, \mathscr{S})=\liminf _{\mathscr{P} \rightarrow \mathscr{S}} \mathscr{E}(\psi, \mathscr{P})
$$

where the convergence is with respect to the Fréchet metric. That $L(\psi, \cdot)$ is lower semi-continuous with respect to Fréchet convergence, and therefore with respect to uniform convergence, follows in the usual manner.

If $k\|\alpha\| \leqq \psi(a, \alpha) \leqq K\|\alpha\|$ and $x \in C(Q, m)$ then it is easy to see that $k L(x) \leqq L(\psi, x) \leqq K L(x)$.

5. Let $\mathscr{D}^{\prime \prime}$ be the collection of all continuous nonnegative valued functions $\bar{\psi}$ on $m \times m$ which are positively homogeneous of degree two, convex, and for which there exists $K \geqq k>0$ such that $k\left[\|a\|^{2}+\|b\|^{2}\right] \leqq \bar{\psi}(a, b) \leqq K\left[\|a\|^{2}+\|b\|^{2}\right]$ for all $(a, b) \in m \times m$.

Let $\bar{\psi} \in \mathscr{D}^{\prime \prime}, c \wedge d \neq 0$, and $\pi$ be the plane determined by $c$ and $d$.

Lemma 5.1. Let $a \neq 0, a \in \pi, \pi_{a}=\{p \in \pi \mid(a \wedge p) /(c \wedge d)>0\}$ and 
$f(p)=\bar{\psi}(a, p) /\|a \wedge p\|$ on $\pi_{a}$. Then the set $E(a)$ of relative minima of $f$ is closed and convex, and $f$ is constant on $E(a)$.

Proof. Suppose that $p$ and $q$ are in $E(a)$ and that $\|a \wedge q\|-$ $\|a \wedge p\|=k>0$. Let $\varphi(w)=\bar{\psi}(a, w p+(1-w) q)$ and $A(w)=$ $\|a \wedge q\|-k w$. Since $\varphi$ is convex and $\varphi(w) / A(w)$ is $(-1 / k)$ times the slope of the line joining $(\|a \wedge q\| / k, 0)$ to $(w, \varphi(w))$, it follows that $(0, \varphi(0)),(1, \varphi(1))$, and $(\|\alpha \wedge q\| / k, 0)$ are collinear and $\varphi / A$ is constant on $[0,1]$. If $k=0$ the convex function $\varphi$ has horizontal tangents at $(0, \varphi(0))$ and at $(1, \varphi(1))$. Hence $\varphi$, and therefore $\varphi / A$, are constant on $[0,1]$.

Now define $g(a)$ to be the value assumed by $f$ on $E(a)$. If $k$ and $K$ are related to $\bar{\psi}$ in the usual way then $k \leqq g(a) \leqq 2 K$.

Lemma 5.2. $g$ is continuous (on $\pi$ ).

Proof. Let $0 \neq a_{n} \rightarrow a \neq 0$ and $g\left(a_{n}\right)=\bar{\psi}\left(a_{n}, p_{n}\right) /\left\|a_{n} \wedge p_{n}\right\|$ where $a_{n}, a$ and $p_{n}$ are all in $\pi$. We can suppose that $p_{n} \rightarrow p_{0}$. Since $g\left(a_{n}\right) \leqq 2 K$ for all $n$, liminf $\left\|a_{n} \wedge p_{n}\right\|>0$. Hence $g(a) \leqq \liminf g\left(a_{n}\right)$. On the other hand, suppose that $g(a)=\bar{\psi}(a, p) /\|a \wedge p\|$. Now $g\left(a_{n}\right) \leqq$ $\bar{\psi}\left(a_{n}, p\right) /\left\|a_{n} \wedge p\right\|$, for sufficiently large $n$, and so limsup $g\left(a_{n}\right) \leqq g(\alpha)$.

Since $g$ is positively homogeneous of degree zero we can define $K(c \wedge d)=\max _{0 \neq a \in \pi} g(a)$.

Now we use $\bar{\psi}$ to generate an area $A \bar{\psi}$ by the formulas

$$
A \bar{\psi}(a \wedge b)=K(a \wedge b)\|a \wedge b\|
$$

and

$$
A \bar{\psi}(\alpha)=\inf _{\Sigma a_{n} \wedge b_{n}=\alpha} \sum A \bar{\psi}\left(a_{n} \wedge b_{n}\right)
$$

LemmA 5.3. If $A \bar{\psi}(a \wedge b)=\bar{\psi}(a, b)$ then $\|a\| / L \leqq\|b\| \leqq\|a\| L$ where $L=\left(K+\sqrt{K^{2}-k^{2}}\right) / k$.

Proof. Let $\|b\|=t^{2}\|a\|$ where $t^{2}>L$. Then $\bar{\psi}(t a, b / t) \leqq$ $K\left(t^{2}\|a\|^{2}+t^{-2}\|b\|^{2}\right)=2 K t^{2}\|a\|^{2}<k\left(1+t^{4}\right)\|a\|^{2} \leqq \bar{\psi}(a, b)$ which is a contradiction.

If $a \wedge b \neq 0$ then by an application of the Hahn-Banach Theorem, [17], there exist $f, g \in m_{1}^{*}$ such that $[a \wedge b, f \wedge g]=\|a \wedge b\|$ and $\max (|f(r)|,|g(r)|) \leqq\|r\| \leqq|f(r)|+|g(r)|$ whenever $r$ is linearly dependent upon $a$ and $b$. Hence $\left(f^{2}(r)+g^{2}(r)\right) / 2 \leqq\|r\|^{2} \leqq 2\left(f^{2}(r)+g^{2}(r)\right)$ for all such $r$ and $\left(\|a\|^{2}+\|b\|^{2}\right) / 4 \leqq\|r\|^{2}+\|s\|^{2} \leqq 4\left(\|a\|^{2}+\|b\|^{2}\right)$ if $r=$ $a \cos \theta-b \sin \theta$ and $s=a \sin \theta+b \cos \theta$.

Let $\mathscr{D}^{\prime}=\left\{\bar{\psi} \in \mathscr{D}^{\prime \prime} \mid \bar{\psi}(a \cos \theta-b \sin \theta, a \sin \theta+b \cos \theta)=\bar{\psi}(a, b)\right.$ for all $\theta, \bar{\psi}=\lim \bar{\psi}_{n}(a, b)$ and $\bar{\psi}_{n} \leqq \bar{\psi}_{n+1}$ where $\left.\bar{\psi}_{n}(a, b)=\bar{\psi}\left({ }_{n} a,{ }_{n} b\right)\right\}$. 
Let $\mathscr{D}$ be the collection of all continuous functions on $m \times m \times m$ for which there exist constants $K \geqq k>0$ such that $k\left(\|b\|^{2}+\|c\|^{2}\right) \leqq$ $\bar{\psi}(a, b, c) \leqq K\left(\|b\|^{2}+\|c\|^{2}\right)$ for all $a, b, c \in m$ and such that $\bar{\psi}_{a} \in \mathscr{D}^{\prime}$, where $\bar{\psi}_{a}(b, c)=\bar{\psi}(a, b, c)$. If $\bar{\psi} \in \mathscr{D}$ then $\bar{\psi}_{n}(a, b, c)=\left(\bar{\psi}_{a}\right)_{n}(b, c)$. We may write $\bar{\psi}_{n}$ for $\bar{\psi}_{n} \mid\left({ }_{n} m \times{ }_{n} m \times{ }_{n} m\right)$. Let $\mathscr{D}_{n}=\left\{\bar{\psi} \in \mathscr{D} \mid \bar{\psi}=\bar{\psi}_{n}\right\}$.

Lemma 5.4. If $\bar{\psi} \in \mathscr{D}^{\prime}$ then $A \bar{\psi}(a \wedge b)=\min \{\bar{\psi}(c, d) \mid c \wedge d=a \wedge b\}$.

The proof is straight-forward.

If $\bar{\psi} \in \mathscr{D}^{\prime \prime}$ then let $N=2(\bar{\psi})^{1 / 2}$.

LEMmA 5.5. Let $\bar{\psi} \in \mathscr{D}^{\prime}$ and $(a, b) \in m \times m$. If there exist $(f, g) \in m^{*} \times m^{*}$ such that $(f, g)$ is a supporting linear functional to (the convex function) $N$ at $(a, b)$ and if $f(a)=g(b)=N(a, b) / 2, f(b)=$ $g(a)=0$, then $A \bar{\psi}(\lambda a+\mu b, \rho a+\sigma b)=[(\lambda a+\mu b) \wedge(\rho a+\sigma b), f \wedge g]$ whenever $\lambda \sigma-\mu \rho \geqq 0$.

Proof. $\quad N(a, \rho a+\sigma b) \geqq(1+\sigma) N(a, b) / 2$ implies that $\bar{\psi}(a, \rho a+\sigma b) \geqq$ $(1+\sigma)^{2} \bar{\psi}(a, b) / 4 \geqq \sigma \bar{\psi}(a, b)$. It follows from Lemma 5.4 that $A \bar{\psi}(a \wedge b)=$ $\bar{\psi}(a, b)$.

LEMMA 5.6. Let $\bar{\psi} \in \mathscr{D}^{\prime}$ and suppose that $A \bar{\psi}(a \wedge b)=\bar{\psi}(a, b)$. Let $\left(f^{\prime}, g^{\prime}\right) \in \pi^{*} \times \sigma^{*}$ satisfy $f^{\prime}(a)=g^{\prime}(b)=N(a, b) / 2, f^{\prime}(b)=g^{\prime}(a)=0$, where $\pi$ is the plane determined by $a$ and $b$. Then $\left(f^{\prime}, g^{\prime}\right)$ is a supporting linear functional to $N \mid(\pi \times \pi)$ at $(a, b)$.

Proof. We write $N$ for $N \mid(\pi \times \pi)$. Let $\lambda, \mu, \rho, \sigma$ be real numbers, $a^{\prime}=\lambda a+\mu b$ and $b^{\prime}=\rho a+\sigma b$. By hypothesis, $\lambda \sigma-\mu \rho>1$ implies $N\left(a^{\prime}, b^{\prime}\right)>N(a, b)$. We must show that $N\left(a^{\prime}, b^{\prime}\right) \geqq(\lambda+\sigma) N(a, b) / 2$ for all $\lambda, \mu, \rho, \sigma$. However, since $N$ is convex, we can suppose that $|\lambda-1|,|\mu|,|\rho|$ and $|\sigma-1|$ are all less than 1/2, which implies that $\lambda \sigma-\mu \rho>0$. Let $\lambda_{n}=(n+\lambda) /(n+1), \mu_{n}=\mu /(n+1), \rho_{n}=\rho /(n+1)$, $\sigma_{n}=(n+\sigma) /(n+1), a_{n}=\lambda_{n} a+\mu_{n} b, b_{n}=\rho_{n} a+\sigma_{n} b$ and $t<(\lambda+\sigma) / 2$. Then $\left(a_{n}, b_{n}\right)=\left(n(a, b)+\left(a^{\prime}, b^{\prime}\right)\right) /(n+1)$ and, for sufficiently large $n$, $\lambda_{n} \sigma_{n}-\mu_{n} \rho_{n}=\left(n^{2}+n(\lambda+\sigma)+\lambda \sigma-\mu \rho\right) /(n+1)^{2}>(n+t)^{2} /(n+1)^{2}$. For such $n, N\left(a_{n}, b_{n}\right)>(n+t) N(a, b) /(n+1)$. Thus $n N(a, b)+$ $N\left(a^{\prime}, b^{\prime}\right)>(n+t) N(a, b)$ and $N\left(a^{\prime}, b^{\prime}\right)>t N(a, b)$. Hence $N\left(a^{\prime}, b^{\prime}\right) \geqq$ $(\lambda+\sigma) N(a, b) / 2$.

Lemma 5.7. Let $\bar{\psi} \in \mathscr{D}^{\prime}$ and suppose that $A \bar{\psi}(a \wedge b)=\bar{\psi}(a, b)$. Then there exist $(f, g) \in m^{*} \times m^{*}$ such that $(f, g)$ is a supporting linear functional to $N$ at $(a, b)$ and $f(a)=g(b)=N(a, b) / 2, f(b)=$ $g(a)=0$. 
This lemma follows from the preceding and the Hahn-Banach Theorem.

If $\bar{\psi} \in \mathscr{D}^{\prime}$ let $S(\bar{\psi})=\{(f, g) \mid(f, g)$ supports $N$ for some $(a, b)$ and $f(a)=g(b)=N(a, b) / 2, f(b)=g(a)=0\}$.

Theorem 5.1. If $\bar{\psi} \in \mathscr{D}^{\prime}$ then

$$
A \bar{\psi}(a \wedge b)=\sup \{[a \wedge b, f \wedge g] \mid(f, g) \in S(\bar{\psi})\} \text {. }
$$

Proof. Suppose that $A \bar{\psi}(a \wedge b)=\bar{\psi}(a, b)$. Then $A \bar{\psi}(a \wedge b)=$ $N^{2}(a, b) / 4=[a \wedge b, f \wedge g]$ for some $(f, g) \in S(\bar{\psi})$, by Lemma 5.7. Now let $(c, d) \in m \times m$. If we choose $\theta$ so that $f(c) \sin \theta+f(d) \cos \theta=0$ and let $c^{\prime}=c \cos \theta-d \sin \theta, \quad d^{\prime}=c \sin \theta+d \cos \theta$, then we obtain $[c \wedge d, f \wedge g]=\left[c^{\prime} \wedge d^{\prime}, f \wedge g\right]=f\left(c^{\prime}\right) g\left(d^{\prime}\right) \leqq\left(f\left(c^{\prime}\right)+g\left(d^{\prime}\right)\right)^{2} / 4$

$$
\leqq N^{2}\left(c^{\prime}, d^{\prime}\right) / 4=\bar{\psi}\left(c^{\prime}, d^{\prime}\right)=\bar{\psi}(c, d)=A \bar{\psi}(c \wedge d) \text {. }
$$

LEMMA 5.8. Let $\bar{\psi}_{0} \in \mathscr{D}^{\prime \prime}$ and $\bar{\psi}(a, b)=\max _{\theta} \bar{\psi}_{0}(a \cos \theta-b \sin \theta$, $a \sin \theta+b \cos \theta)$. Then $A \bar{\psi}_{0}=A \bar{\psi}$.

Proof. Let $a^{\prime} \wedge b^{\prime} \neq 0$. There exist $a_{1}$ and $b_{1}$ with $a_{1} \wedge b_{1}=$ $a^{\prime} \wedge b^{\prime}$ such that $A \bar{\psi}\left(a_{1} \wedge b_{1}\right)=\bar{\psi}\left(a_{1}, b_{1}\right)$ and there exists $\theta$ such that $\bar{\psi}\left(a_{1}, b_{1}\right)=\bar{\psi}_{0}(a, b)$ where $a=a_{1} \cos \theta-b_{1} \sin \theta$ and $b=a_{1} \sin \theta+b_{1} \cos \theta$. Let $\pi$ be the plane determined by $a^{\prime}$ and $b^{\prime}$ and $N_{0}=2\left(\bar{\psi}_{0}\right)^{1 / 2}$. If both $N_{0} \mid(\pi \times \pi)$ and $N \mid(\pi \times \pi)$ have unique supporting linear functionals at $(a, b)$ they must coincide since $N \geqq N_{0}$ and $N(a, b)=N_{0}(a, b)$. Thus there exist $f^{\prime}$ and $g^{\prime}$ on $\pi$ such that $\left(f^{\prime}, g^{\prime}\right)$ is the supporting linear functional and $f^{\prime}(a)=g^{\prime}(b)=N(a, b) / 2, \quad f^{\prime}(b)=g^{\prime}(a)=0$. Thus $N_{0}(a, \rho a+\sigma b) \geqq\left(f^{\prime}(a)+\sigma g^{\prime}(b)\right)^{2}=(1+\sigma)^{2} N^{2}(a, b) / 4$ whenever $\sigma>0$. Hence $A \bar{\psi}_{0}(a \wedge b)=\bar{\psi}_{0}(a, b)=\bar{\psi}(a, b)=A \bar{\psi}(a \wedge b)$. If either $N_{0} \mid(\pi \times \pi)$ or $N \mid(\pi \times \pi)$ does not have a unique supporting linear functional at $(a, b)$, let $\varepsilon>0$ and choose $N_{0}^{\prime}$ on $\pi \times \pi$ to be strictly convex, of class $C^{\prime \prime}$, positively homogeneous of degree one and such that $N_{0} \leqq$ $N_{0}^{\prime}<(1+\varepsilon)^{2} N_{0}$. Let $\bar{\psi}_{0}^{\prime}=N_{0}^{\prime 2} / 4$ and $\bar{\psi}^{\prime}$ be defined for $\bar{\psi}_{0}^{\prime}$ as $\bar{\psi}$ was for $\bar{\psi}_{0}$. Then $A \bar{\psi} \leqq A \bar{\psi}^{\prime}=A \bar{\psi}_{0}^{\prime}<(1+\varepsilon) A \bar{\psi}_{0}$.

TheOREM 5.2. Let $M$ be a norm on $m$ and $\bar{\psi}(a, b)=\left(M^{2}(a)+\right.$ $\left.M^{2}(b)\right) / 2$. If $M^{*}(f)=\sup \{f(a) \mid M(a)=1\}$ for all $f \in m^{*}$ then $A \bar{\psi}=\psi$ where $\psi(a \wedge b)=\sup \left\{[a \wedge b, f \wedge g] \mid M^{*}(f)=M^{*}(g)=1\right\}$.

Proof. Suppose that $a \wedge b \neq 0$. Then, by the argument of [17] where $M=\|\|$, there exist $c, d \in m$ and $f, g \in m^{*}$ such that $M^{*}(f)=$ $M^{*}(g)=1, c \wedge d=a \wedge b, f(c)=M(c)=M(d)=g(d), f(d)=g(c)=0$, and $\psi(a \wedge b)=[a \wedge b, f \wedge g]$. It follows that $(f, g) \in S(\bar{\psi})$ and so $A \bar{\psi} \geqq \psi$. On the other hand, if $(f, g) \in S(\bar{\psi})$ then there exists $a$ and 
$b$ with $a \wedge b \neq 0$ such that $(f, g)$ supports $N$ at $(a, b)$ and $f(a)=$ $g(b)=N(a, b) / 2, \quad f(b)=g(a)=0 . \quad$ Since $\bar{\psi}(t a, b / t)$ has a relative minimum at one, we see that $M(a)=M(b)$. Now let $c \in m$, and choose $t>0$ so that $M(t b)=M(c)$. We have $f(c)+M(c)=f(c)+$ $g(t b) \leqq 2\left(\left(M^{2}(c)+M^{2}(t b) / 2\right)^{1 / 2}=2 M(c)\right.$. Thus $M^{*}(f) \leqq 1$ and, similarly, $M^{*}(g) \leqq 1$ and so $\psi \geqq A \bar{\psi}$.

6. If $x$ is a $D$-map on $J$ then $\left\{x_{u}^{i}\right\}$ is defined almost everywhere in Int $J$ and is an element of $m$. We define $x_{u}=\left\{x_{u}^{i}\right\}$ and $x_{v}=\left\{x_{v}^{i}\right\}$ [21]. Let $D(x)=\iint\left(\left\|x_{u}\right\|^{2}+\left\|x_{v}\right\|^{2}\right), \quad I(\psi, x)=\iint \psi\left(x, x_{u} \wedge x_{v}\right)$, and $I(\bar{\psi}, x)=\iint \bar{\psi}\left(x, x_{u}, x_{v}\right)$ where all of the integrals are taken over Int $J$.

Suppose that $\left\{x_{n}\right\}$ is a sequence of continuous functions on the unit circle $\mathscr{C}$. Then $\left\{x_{n}\right\}$ satisfies the three point condition if there is a $\delta>0$ and $w_{i n} \in \partial \mathscr{C}, i=1,2,3$, such that $\left\|w_{i n}-w_{j_{n}}\right\|>\delta$, $\left\|x_{n}\left(w_{i n}\right)-x_{n}\left(w_{j_{n}}\right)\right\|>\delta$ whenever $i \neq j, i, j=1,2,3$.

If $\bar{\psi} \in \mathscr{D}_{n}$, if $x_{k}$ and $x$ are $D$-maps on $\mathscr{C}$ and if $x_{k}^{i}$ converges uniformly to $x^{i}$ for each $i$, then $I(\bar{\psi}, x) \leqq \liminf I\left(\bar{\psi}, x_{k}\right)$ [13]. Hence $I(\bar{\psi}, x) \leqq \liminf I\left(\bar{\psi}, x_{k}\right)$ for all $\bar{\psi} \in \mathscr{D}$.

If $x$ is continuous on a Jordan region $J$ into a Banach space $B$ and if $\mathscr{S}$ is the Fréchet surface determined by $x$ then $\partial \mathscr{S}$ is the Fréchet curve represented by $x \mid \partial J$.

The proof of the following lemma is modeled after a proof in [2].

LEMma 6.1. Let $\bar{H} \in \mathscr{D}$ where $B=R_{n}$ and suppose that $\bar{H}$ is of class $C^{\prime \prime}$ and strictly convex in its last two arguments. Let $\mathscr{P}$ be an open non-degenerate polyhedron in $B$. Let $p_{i}$ and $q_{i}, i=1,2,3$, be distinct points of $\partial C$ and $\partial \mathscr{P}$, respectively. Then there exists $a$ $D$-map $x^{*}$ on $\mathscr{C}$ which represents $\mathscr{P}$ such that $x^{*}\left(p_{i}\right)=q_{i}$ and. $I\left(\bar{H}, x^{*}\right)=I\left(A \bar{H}, x^{*}\right)$.

Proof. We mention, first, two properties of $\bar{H}$. If $y$ is a $D$-map. and $T$ is a conformal transformation, then $I(\bar{H}, y)=I(\bar{H}, T y)$. Also, $\sum_{i} y_{v}^{i} \bar{H}_{i},\left(y, y_{u}, y_{v}\right)=\sum_{i} y_{u}^{i} H_{, i} i\left(y, y_{w}, y_{v}\right)$ where $\bar{H}_{, i}=\left(\partial / \partial y_{u}^{i}\right) \bar{H}$ and $\bar{H}_{, i}=$ $\left(\partial / \partial y_{v}^{i}\right) \bar{H}$.

Let $K$ be the nonempty class of all representations of $\mathscr{P}$ on $\mathscr{C}$ which are $D$-maps. Let $\bar{I}=\inf _{x \in K} I(\bar{H}, x)$ and let $\left\{x_{n}\right\}$ be a minimizing sequence in $K$. Since $I\left(\bar{H}, x_{n}\right)=I\left(\bar{H}, T x_{n}\right)$ for $T$ as above, we can suppose that $x_{n}\left(p_{i}\right)=q_{i}$. By Theorems 5 and 6 [21], the sequence $\left\{x_{n}\right\}$ is equicontinuous. By deleting some terms, if necessary, we can suppose that $x_{n}$ converges uniformly to $x^{*}$ where $x^{*} \in \mathscr{P}$ and $x^{*}$ is a. $D$-map [21; Th. 3]. Thus $x^{*} \in K$ and $I\left(\bar{H}, x^{*}\right)=\bar{I}$. 
Now let $\varphi$ and $\psi$ be Lipschitzian with constant $M$ on $\mathscr{C}$. The transformation $U(u, v)=(\alpha, \beta), \quad \alpha=u+\varepsilon \varphi(u, v), \quad \beta=v+\varepsilon \psi(u, v)$, together with its inverse, is Lipschitzian if $|\varepsilon|<1 /(3 M)$. Set $x(\alpha, \beta)=$ $x^{*}(u, v)$. Then $x$ is a $D$-map. Let $T$ be the conformal transformation of domain $x$ onto $\mathscr{C}$ which takes $U\left(p_{i}\right)$ onto $p_{i}$ and put $X=x T^{\vee}$. Then $X \in K$. Put

$$
J(\varepsilon)=I(\bar{H}, X)=\iint_{\mathscr{C}} D^{-1} \bar{H}\left(x^{*}, x_{u}^{*} \beta_{v}-x_{v}^{*} \alpha_{u},-x_{u}^{*} \alpha_{v}+x_{v}^{*} \alpha_{u}\right) d u d v
$$

where $D=\partial(\alpha, \beta) / \partial(u, v)$. Then

$$
\begin{aligned}
0= & 2 J^{\prime}(0)= \\
& \iint_{\mathscr{C}}\left\{\left(\left(G^{*}-E^{*}\right) \varphi_{u}-2 F^{*} \varphi_{v}\right)+\left(\left(E^{*}-G^{*}\right) \psi_{v}-2 F^{*} \psi_{u}\right)\right\} d u d v
\end{aligned}
$$

where

$$
\begin{gathered}
E^{*}=\sum_{i} x_{u}^{i *} \bar{H}_{i},\left(x^{*}, x_{u}^{*}, x_{v}^{*}\right), \\
F^{*}=\sum_{i} x_{u}^{i *} \bar{H}_{, i}\left(x^{*}, x_{u}^{*}, x_{v}^{*}\right)=\sum_{i} x_{v}^{i *} \bar{H}_{i},\left(x^{*}, x_{u}^{*}, x_{v}^{*}\right)
\end{gathered}
$$

and

$$
G^{*}=\sum_{i} x_{v}^{i *} \bar{H}_{, i}\left(x^{*}, x_{u}^{*}, x_{v}^{*}\right)
$$

Since $\varphi$ and $\psi$ are arbitrary, we obtain

$$
\iint_{\mathscr{C}}\left(-A \varphi_{u}+B \varphi_{v}\right)=0 \text { and } \iint_{\mathscr{C}}\left(A \psi_{v}+B \psi_{u}\right)=0
$$

where $A=E^{*}-G^{*}$ and $B=-2 F^{*}$. By (1) and Haar's lemma [13]

$$
\int_{\partial R}(A d v-B d u)=0
$$

for almost all rectangles $R \subset \mathscr{C}$. For each $h>0$ let

$$
\mathscr{C}_{h}=\{(u, v) \in \mathscr{C} \mid[u-h, u+h] \times[v-h, v+h] \subset \mathscr{C}\}
$$

and let $A_{h}$ and $B_{h}$ be the $h$-average functions of $A$ and $B$ defined on $\mathscr{C}_{h}$. These functions are continuous and satisfy (2) for every rectangle $R \subset \mathscr{C}_{h}$. Let $\zeta_{h}(u, v)=\int_{(0,0)}^{(u, v)}\left(A_{h} d v-B_{h} d u\right)$, the integral being independent of the (rectifiable) path joining $(0,0)$ to $(u, v)$. Now $\zeta_{n u}=$ $-B_{h}$ and $\zeta_{h v}=A_{h}$. Using the other part of (1) in a similar fashion we obtain $\eta_{h u}=-A_{h}$ and $\eta_{h v}=-B_{h}$. Thus $\zeta_{h}$ and $\eta_{h}$ are harmonic on $\mathscr{C}_{h}$. By [13; Th. 4.2, p. 74], $\zeta_{h}$ and $\eta_{h}$ both vanish on $\partial \mathscr{C}_{h}$. Thus 
$E^{*}=G^{*}$ and $F^{*}=0$ almost everywhere in $\mathscr{C}$, and by Lemma 5.5, $A \bar{H}\left(x^{*}, x_{u}^{*} \wedge x_{v}^{*}\right)=\bar{H}\left(x^{*}, x_{u}^{*}, x_{v}^{*}\right)$ wherever these equalities hold.

If $\bar{\psi} \in \mathscr{D}$ and $x$ is a $D$-map on an open set $G$ then $x$ is $A \bar{\psi}$-quasiconformal on $G$ if $\bar{\psi}\left(x, x_{u}, x_{v}\right)=A \bar{\psi}\left(x, x_{u} \wedge x_{v}\right)$ almost everywhere on $G$. If $J$ is a Jordan region then we say that $x$ is $A \bar{\psi}$-quasi-conformal on $J$ rather than on Int $J$.

LEMMA 6.2. Let $\mathscr{P}$ be an open non-degenerate polyhedron and suppose that $\bar{\psi} \in \mathscr{D}$. If $p_{i}$ and $q_{i}, i=1,2,3$, are distinct points of $\partial \mathscr{C}$ and $\partial \mathscr{P}$, respectively, then $\mathscr{P}$ has an $A \bar{\psi}$-quasi-conformal representation $x$ on $\mathscr{C}$ such that $x\left(p_{i}\right)=q_{i}$.

Proof. There exist $\bar{H}_{n}$ as in the last lemma with $\bar{H}_{n} \leqq \bar{H}_{n+1}$ and $\bar{\psi}=\lim \bar{H}_{n}$. By Dini's Theorem, we can suppose that $\bar{\psi}<(1+1 / n) \bar{H}_{n}$ on $|\mathscr{P}| \times B_{1} \times B_{1}$, where $|\mathscr{P}|$ is the compact set covered by $\mathscr{P}$. Let $x_{n} \in \mathscr{P}$ be $A \bar{H}_{n}$-quasi-conformal with $x_{n}\left(p_{i}\right)=q_{i}$. Then, as before, there exists a $D$-map $x \in \mathscr{P}$ and we can suppose that $x_{n}$ converges uniformly to $x$. Hence $I(\bar{\psi}, x) \leqq \liminf I\left(\bar{\psi}, x_{n}\right) \leqq \liminf (1+1 / n) I\left(\bar{H}, x_{n}\right)=$ $\liminf (1+1 / n) I\left(A H_{n}, x_{n}\right)=\liminf (1+1 / n) I\left(A H_{n}, x\right) \leqq I(A \bar{\psi}, x)$.

THEOREM 6.1. If $\mathscr{S}$ is an open non-degenerate surface of finite Lebesgue area, if $\bar{\psi} \in \mathscr{D}$, and if $p_{i}$ and $q_{i}, i=1,2,3$, are distinct points of $\partial \mathscr{C}$ and $\partial \mathscr{S}$, then $\mathscr{S}$ has an $A \bar{\psi}$-quasi-conformal representation $x$ on $\mathscr{C}$ such that $x\left(p_{i}\right)=q_{i}$.

Proof. There exist open non-degenerate polyhedra $\mathscr{P}_{n} \rightarrow \mathscr{S}$ such that $\mathscr{E}\left(A \bar{\psi}, \mathscr{P}_{n}\right) \rightarrow L(A \bar{\psi}, \mathscr{S})$. Let $q_{i, n}, i=1,2,3$, be distinct points of $\partial \mathscr{P}_{n}$ such that $q_{i, n} \rightarrow q_{i}$. There exist $A \bar{\psi}$-quasi-conformal representations $x_{n}$ of $\mathscr{P}_{n}$ such that $x_{n}\left(p_{i}\right)=q_{i, n}$. Hence, as before, there exists a $D$-map $x \in \mathscr{S}$ and we can suppose that $x_{n}$ converges uniformly to $x$. Thus

$$
\begin{aligned}
I(\bar{\psi}, x) & \leqq \liminf I\left(\bar{\psi}, x_{n}\right)=\liminf I\left(A \bar{\psi}, x_{n}\right)=\liminf \mathscr{E}\left(A \bar{\psi}, \mathscr{P}_{n}\right) \\
& =L(A \bar{\psi}, \mathscr{S})=I(A \bar{\psi}, x) .
\end{aligned}
$$

7. A subset $\mathscr{K}$ of $m$ is c-closed if $a \in \mathscr{K}$ whenever there exists $\left\{a_{n}\right\}$ in $\mathscr{K}$ such that $a_{n}^{i} \rightarrow a^{i}$ for each $i$. If $\mathscr{K} \cap S$ is $c$-closed for each sphere $S$ then $\mathscr{K}$ is locally c-closed. Evidently $\mathscr{K}$ is locally $c$-closed if $\mathscr{K}$ is locally compact.

If $\mathscr{S}$ is a Fréchet surface of the type of a two-cell, let $\partial \mathscr{S}$ be the Fréchet curve defined by $x \mid \partial \mathscr{C}$ where $x$ is a representation of $\mathscr{S}$ on $\mathscr{C}$. Let $d\left(\gamma_{1}, \gamma_{2}\right)$ be the Fréchet distance between the Fréchet curves $\gamma_{1}$ and $\gamma_{2}$, each of the type of the circle.

If $\mathscr{K}$ is a convex locally $c$-closed subset of $m$, if $\gamma$ is a simple 
closed curve in $\mathscr{C}$ and if $\psi \in \mathscr{A}$ then

$$
m(\psi, \mathscr{K}, \gamma)=\liminf _{d(\partial \mathscr{P}, \gamma) \rightarrow 0} \mathscr{E}(\psi, \mathscr{P})
$$

and

$$
a(\psi, \mathscr{K}, \gamma)=\inf _{\partial \mathscr{S}=\gamma} L(\psi, \mathscr{S})
$$

where $\mathscr{P}$ is a polyhedron and $\mathscr{S}$ is a surface, each of the type of the disc, and each is contained in $\mathscr{K}$. By Lemma 3.4 we can suppose that each $\mathscr{P}$ in the definition of $m(\psi, \mathscr{K}, \gamma)$ is open non-degenerate.

Let $T(\mathscr{K})$ be the set of all $D$-maps on $\mathscr{C}$ with range in $\mathscr{K}$ and $T(\mathscr{K}, \gamma)=\{x \in T(\mathscr{K})|x| \partial \mathscr{C} \in \gamma\}$.

Lemma 7.1. Let $\mathscr{S}$ be contained in $\mathscr{K}$. Then there exists a sequence of polyhedra $\left\{\mathscr{P}_{n}\right\}$ contained in $\mathscr{K}$ with $\mathscr{P}_{n} \rightarrow \mathscr{S}$ and $\mathscr{E}\left(\psi, \mathscr{P}_{n}\right) \rightarrow L(\psi, \mathscr{S})$.

Proof. Let us suppose that $\{a \in m \mid\|a\|<\delta\} \subset \mathscr{K}$ for some $\delta>0$. If $\mathscr{T}$ is a surface with representation $x$ and if $\rho$ is a positive number, let $\rho \mathscr{T}$ be the surface determined by $\rho x$. Now suppose that $\left\{\mathscr{Q}_{n}\right\}$ is a sequence of polyhedra with $\mathscr{Q}_{n} \rightarrow \mathscr{S}$ and $\mathscr{E}\left(\psi, \mathscr{Q}_{n}\right) \rightarrow L(\psi, \mathscr{S})$. Then $\left\{\mathscr{P}_{n}\right\}$ can be chosen by $\mathscr{P}_{n}=\left(\delta \mathscr{Q}_{n}\right) /\left(\delta+2 d\left(\mathscr{Q}_{n}, \mathscr{S}\right)\right)$. If $\mathscr{K}$ has an interior point other than 0 , a translation reduces the problem to the preceding. If $\mathscr{K}$ has no interior point, let $B$ be the space spanned by $\mathscr{K}$ and let $L_{B}(\psi, \mathscr{S})$ be the area defined by restricting sequences of polyhedra approximating $\mathscr{S}$ to be in $B$. Since $L_{B}(\psi, \mathscr{S})=L(\psi, \mathscr{S})$ as in [17] and the argument applies to $L_{B}$, the lemma is proved.

Now suppose $\mathscr{S}$ is contained in $\mathscr{K}$ and $\partial \mathscr{S}=\gamma$. There exists a sequence $\mathscr{P}_{n}$ of polyhedra contained in $\mathscr{K}$ such that $\mathscr{E}\left(\psi, \mathscr{P}_{n}\right) \rightarrow$ $L(\psi, \mathscr{S})$. Hence, [12], $m(\psi, \mathscr{K}, \gamma) \leqq \liminf \mathscr{E}\left(\psi, \mathscr{P}_{n}\right)=L(\psi, \mathscr{S})$ and, consequently, $m(\psi, \mathscr{K}, \gamma) \leqq a(\psi, \mathscr{K}, \gamma)$.

If $G$ is an open connected set in the plane, if $x=\left\{x^{i}\right\}$ where each $x^{i}$ is of class $\mathfrak{I}_{2}$ on $G$ and if $D(x)$ is finite, then $x$ is of class $\mathscr{P}_{2}$ on $G$ [13]. If $G$ is of class $K$, in particular, if $G$ is the interior of a circle, or the intersection of the interiors of two circles, then there exists a function $\varphi$ on $\partial G$ which plays the role of the boundary value function for $x$. We shall write $x \mid \partial G$ for $\varphi$ and $x(p)$ for $\varphi(p)$ for $p \in \partial G$.

Let $x$ be of class $\mathscr{P}_{2}$ on Int $\mathscr{C}$. Then $z$ is a simple cone-function for $x$ on Int $\mathscr{C}$ if

$$
z(p)=\left\{\begin{array}{cl}
\|p\| x(p /\|p\|)+(1-\|p\|) q, & p \neq(0,0) \\
q & , \quad p=(0,0)
\end{array}\right.
$$

whenever $q$ is contained in the convex hull of $x(\partial \dot{C})$. Now let $x$ be 
of class $\mathscr{P}_{2}$ on a region $J$ of class $K$. Then $z$ is a cone-function for $x$ on $J$ if there exists a conformal transformation $T$ from $J$ onto $\mathscr{C}$ such that $z=w T$ and $w$ is a simple cone-function for $x T^{\vee}$. By some remarks in $\S 5$,

$$
D(w) / 4 \leqq D(z) \leqq 4 D(w) .
$$

We require some slight modification of one of Morrey's results. Let $C(P, r)$ be the open circle with center $P$ and radius $r$.

Lemma 7.2. Let $x$ be class $\mathscr{P}_{2}$ on $C(0, R)$ with $D(x)=M<\infty$. Suppose there is a number $k>0$ such that

$$
D(x \mid C(0, r)) \leqq k D(H(x, r)), \quad 0<r \leqq R,
$$

whenever $H(x, r)$ is a simple cone-function for $x$ over $C(0, r)$. Then

$$
D(x \mid C(0, r)) \leqq M(r / R)^{1 /(6 k)}, \quad 0 \leqq r \leqq R .
$$

We use polar coordinates and let

$$
\psi(r)=D(x \mid C(0, r))=\int_{0}^{r} \rho\left\{\int_{0}^{2 \pi}\left\|x_{\rho}(\rho, \theta)\right\|^{2}+\rho^{-2}\left\|x_{\theta}(\rho, \theta)\right\|^{2} d \theta\right\} d \rho .
$$

Since $\psi$ is absolutely continuous on $[0, R]$,

$$
r \psi^{\prime}(r)=\int_{0}^{2 \pi}\left[r^{2}\left\|x_{\rho}(r, \theta)\right\|^{2}+\left\|x_{\theta}(r, \theta)\right\|^{2}\right] d \theta
$$

for almost all $r$ in $[0, R]$.

Now we compute

$$
\begin{aligned}
& D(H(x, r))=r^{-2} \int_{0}^{r} \int_{0}^{2 \pi} \rho\left[\|x(r, \theta)\|^{2}+\left\|x_{\theta}(r, \theta)\right\|^{2}\right] d \rho d \theta \\
& \quad \leqq r^{-2} \int_{0}^{r} \int_{0}^{2 \pi} \rho\left[2^{-1} \int_{0}^{2 \pi}\left\|x_{\theta}(r, \varphi)\right\| d \varphi\right]^{2} d \rho d \theta+r^{-2} \int_{0}^{r} \int_{0}^{2 \pi} \rho\left\|x_{\theta}(r, \theta)\right\|^{2} d \rho d \theta \\
& \quad \leqq \frac{\pi r^{-2}}{2} \int_{0}^{r} \int_{0}^{2 \pi} \rho \int_{0}^{2 \pi}\left\|x_{\theta}(r, \varphi)\right\|^{2} d \varphi d \rho d \theta+2^{-1} \int_{0}^{2 \pi}\left\|x_{\theta}(r, \theta)\right\|^{2} d \theta \\
& \quad \leqq \frac{\pi^{2}+1}{2} \int_{0}^{2 \pi}\left\|x_{\theta}(r, \varphi)\right\|^{2} d \varphi \leqq 6 r \psi^{\prime}(r)
\end{aligned}
$$

for almost all $r$, where we have made use of the fact that $\operatorname{diam} x(\partial \mathscr{C}) \leqq$ (length $x \mid \partial \mathscr{C}$ ) $/ 2$.

Thus $\psi(r) \leqq 6 k r \psi^{\prime}(r)$ and $\left(r^{-1 /(6 k)} \psi\right)^{\prime} \geqq 0$.

The following lemma is usually stated for surfaces in Euclidean space, but the proof, with trivial modifications, shows that it is true for surfaces in $m$.

LEMma 7.3. Let $\left\{\mathscr{S}_{n}\right\}$ be a sequence of Fréchet surfaces such that $d\left(\partial \mathscr{S}_{n}, \gamma\right) \rightarrow 0$ where $\gamma$ is a simple closed curve. Suppose that $x_{n}$ is 
a D-representation of $\mathscr{S}_{n}$ on $\mathscr{C}$ such that $\left\{x_{n}\right\}$ satisfies the three point condition and such that $\left\{D\left(x_{n}\right)\right\}$ is uniformly bounded. Then $\left\{x_{n} \mid \partial \mathscr{C}\right\}$ is equicontinuous.

THEOREM 7.1. If $T(\mathscr{K}, \gamma)$ is not empty and if $\bar{\psi} \in \mathscr{D}$ then there exists $x \in T(\mathscr{K}, \gamma)$ such that $I(\bar{\psi}, x)=\inf \{I(\bar{\psi}, y) \mid y \in T(\mathscr{K}, \gamma)\}$. In addition, $x$ is open non-degenerate, $A \bar{\psi}$-quasi-conformal and can be chosen to take three distinct points of $\partial \mathscr{C}$ into three distinct points of $\gamma$.

Proof. If $\mathscr{K}$ were a finite dimensional subspace of $m$ then the first statement would follow from the remarks [13; p. 45]. The proof there is sufficient to permit $\mathscr{K}$ to be a convex subset of a finite dimensional space. This last condition may be deleted by replacing [13; Th. 6.1] with Lemma 7.2. This part of the proof is essentially the proof required for Theorem 7.2, and it is outlined after the statement of that theorem.

If $x$ were not open non-degenerate, there would exist a retraction $y$ of $x$, thus $y \in T(\mathscr{K}, \gamma)$, which would be open non-degenerate. By the representation theorem there would exist an $A \bar{\psi}$-quasi-conformal conformal map $z$, Fréchet equivalent to $y$ and taking three distinct points of $\partial \mathscr{C}$ into three distinct points of $\gamma$, and we would have $I(\bar{\psi}, z)=I(A \bar{\psi}, z)=I(A \bar{\psi}, y)<L(A \bar{\psi}, x)=I(A \bar{\psi}, x) \leqq I(\bar{\psi}, x)$.

Similarly, the assumption that $x$ is not $A \bar{\psi}$-quasi-conformal leads to a contradiction.

The theorem shows that there exists an open non-degenerate surface $\mathscr{S}$ and a representation $x$ of $\mathscr{S}$ which is $A \bar{\psi}$-quasi-conformal such that $L(A \bar{\psi}, \mathscr{S})=a(A \bar{\psi}, \mathscr{K}, \gamma)$. Furthermore, under suitable conditons on $\bar{\psi}, x$ has certain differentiability properties [13; Ch. VII].

TheOREm 7.2. Let $\bar{\psi} \in \mathscr{D}$. If $m(A \bar{\psi}, \mathscr{K}, \gamma)<\infty$ then there exists an open non-degenerate surface $\mathscr{S}$ with an $A \bar{\psi}$-quasi-conformal representation $x$ on $\mathscr{C}$ such that $x \in T(\mathscr{K}, \gamma)$ and $a(A \bar{\psi}, \mathscr{K}, \gamma)=$ $m(A \bar{\psi}, \mathscr{K}, \gamma)=L(A \bar{\psi}, \mathscr{S})$.

Proof. Let $\left\{\mathscr{P}_{n}\right\}$ be a sequence of open non-degenerate polyhedra in $\mathscr{K}$ with $\partial \mathscr{P}_{n} \rightarrow \gamma$ and $\mathscr{E}\left(A \bar{\psi}, \mathscr{P}_{n}\right) \rightarrow m(A \bar{\psi}, \mathscr{K}, \gamma)=I$. Then there exist $A \psi$-quasi-conformal representations $y_{n}$ of $\mathscr{P}_{n}$ such that $\left\{y_{n}\right\}$ satisfies the three point condition and $I\left(\bar{\psi}, y_{n}\right)<I+1$, for sufficiently large $n$. Thus $\left\{D\left(y_{n}\right)\right\}$ is uniformly bounded and, by deleting some terms if necessary, there is a function $y$ such that $y_{n}^{i}$ converges weakly in $\Omega_{2}$ to $y^{i}$. Since $I(\bar{\psi}, \cdot)$ is lower semicontinuous with respect to this convergence, $I(\bar{\psi}, y)=I$ and thus $D(y)$ is finite and $y$ is in $\mathscr{P}_{2}$. That there exists $k>0$ for which $D(x \mid J) \leqq k D(H(x, J))$, and $H(x, J)$ is 
a cone-function for $x$ over $J$, follows as in [13; $p$. 45]. With only trivial changes, [13; p. 13, Th. 2.1] holds in our situation and it follows that $y$ is continuous on each closed subset of Int $\mathscr{C}$. That $y$ is continuous on a neighborhood of $\partial \mathscr{C}$ is proved as in [13; pp. 43-44] except that we must replace the harmonic functions used there by conefunctions. Thus $y$ is continuous on $\mathscr{C}$ and is in $\mathscr{P}_{2}$, that is, $y$ is a $D$-map. That range $y \subset \mathscr{K}$ and $y \mid \partial \mathscr{C} \in \mathcal{Y}$ follows from the fact that range $y$ is contained in a suitably large sphere and $\mathscr{K}$ is locally $c$ closed. Hence $y \in T(\mathscr{K}, \gamma)$.

It is obvious that $y$ is open non-degenerate. Hence there exists an $A \bar{\psi}$-quasi-conformal function $z$ which is Fréchet equivalent to $y$ and takes three distinct points of $\partial \mathscr{C}$ into three distinct points of $\gamma$. By Theorem 7.1 there exists $x \in T(\mathscr{K}, \gamma)$ taking the same three points into the proper image points such that

$$
\begin{aligned}
I(\bar{\psi}, x) & =\inf \{I(\bar{\psi}, w) \mid w \in T(\mathscr{K}, \gamma\} \leqq I(\bar{\psi}, z)=I(A \bar{\psi}, z)=I(A \bar{\psi}, y) \\
& =m(A \bar{\psi}, \mathscr{K}, \gamma) \leqq a(A \bar{\psi}, \mathscr{K}, \gamma) \leqq I(A \bar{\psi}, x) \leqq I(\bar{\psi}, x) .
\end{aligned}
$$

Let $\mathscr{S}$ be the surface determined by $x$.

Since each surface can be approximated arbitrarily closely in both the Frechet metric and in $A \bar{\psi}$-Lebesgue area, by polyhedra, it follows that $m(A \bar{\psi}, \mathscr{K}, \gamma) \leqq \lim \inf L\left(A \bar{\psi}, \mathscr{S}_{n}\right)$ whenever $\left\{\mathscr{S}_{n}\right\}$ is a sequence of surfaces in $\mathscr{K}$ such that $d\left(\partial \mathscr{S}_{n}, \gamma\right) \rightarrow 0$.

COROLLARY. Let $\left\{\gamma_{n}\right\}$ be a sequence of Jordan curves in $\mathscr{K}$ with $d\left(\gamma_{n}, \gamma\right) \rightarrow 0$. Then $m(A \bar{\psi}, \gamma) \leqq \liminf m\left(A \bar{\psi}, \mathscr{K}, \gamma_{n}\right)$.

\section{REFERENCES}

1. S. Banach, Théorie des operations linéaires, Warsaw, 1932.

2. L. Cesari, Criteri di uguale continuità ed applicazioni alla quadratura delle superficie, Ann. Scuola Norm. Sup. Pisa (2) 12 (1943), 61-84.

3. - Surface area, Princeton, 1956.

4. - An existence theorem of calculus of variations for integrals on parametric surfaces, Amer. J. Math. 74 (1952), 265-295.

5. — Su un particulare processo di retrazione per. superficie, Riv. Mat. Univ. Parma 1 (1950), 19-44.

6. - Condizioni sufficienti per la semicontinuità degli integrali sopra una superficie in forma parametrica, Ann. Scuola Norm. Sup. Pisa (2) 12 (1943), 61-84.

7. J. M. Danskin, On the existence of minimizing surfaces in parametric double integral problems of the calculus of variations, Riv. Mat. Univ. Parma, 3 (1952), 43-63.

8. H. Federer, Essential multiplicity and Lebesgue area, Proc. Nat Acad. Sci. USA, 34 (1948), 611-616.

9. W. H. Fleming, Nondegenerate surfaces of finite topological type, Trans. Amer. Math. Soc., 10 (1959), 323-335.

10. E. J. McShane, Parametrization of saddle surfaces with applications to the problem of Plateau, Trans. Amer. Math. Soc., 35 (1933), 716-733. 
11. Existence theorems for double integral problems of calculus of variations, Trans. Amer. Math. Soc., 38 (1935), 549-563.

12. - Extension of range of functions, Bull. Amer. Soc., 40 (1934), 837-842.

13. C. B. Morrey, Jr., Multiple integral problems in the calculus of variations and related topics, University of California, 1943, 1-130.

14. - An analytic characterization of surfaces of finite Lebesgue area, Amer. J. Math., 57 (1935), 692-702.

15. - The parametric variational problem for double integrals, Communications in Pure and Applied Mathematics 14 (1961), 569-575.

16. A. C. Sigalov, Two-dimensional problems of the calculus of variations, Uspehi Matem. Nauk (N.S.) 6, 42 (1951), 16-101.

17. E. Silverman, Definitions of Lebesgue area for surfaces in metric spaces, Riv. Mat. Univ. Parma 2 (1951), 47-76.

18. — An intrinsic property of Lebesgue area, Riv. Mat. Univ. Parma 2 (1951), 195-201.

19. —_ An intrinsic inequality for Lebesgue area, Pacific Math., 6 (1956), 363-372.

20. - Set functions associated with Lebesgue area, Pacific J. Math., 2 (1952), 243-250.

21. - Morrey's representation theorem for surfaces in metric spaces, Pacific J. Math., 7 (1957), 1666-1690.

22. - The equivalence of two extensions of Lebesgue area, Proc. Amer. Math. Soc., 9 (1958), 971-672.

23. L. H. Turner, The direct method in the calculus of variations, Thesis, Purdue Univ., 1957.

24. J. W. T. Youngs, The representation problem for Fréchet surfaces, Amer. Math. Soc. Memoirs, 8, 1951. 



\section{PACIFIC JOURNAL OF MATHEMATICS}

\section{EDITORS}

Robert Osserman

Stanford University

Stanford, California

M. G. Arsove

University of Washington

Seattle 5 , Washington
J. DugundjI

University of Southern Califorma: Los Angeles 7, California

Lowell J. Paige

University of California

Los Angeles 24, California

\section{ASSOCIATE EDITORS}

E. F. BECKENBACH

B. H. NeumanN

F. WOLF

K. YosIDA

\section{SUPPORTING INSTITUTIONS}

UNIVERSITY OF BRITISH COLUMBIA

CALIFORNIA INSTITUTE OF TECHNOLOGY

UNIVERSITY OF CALIFORNIA

MONTANA STATE UNIVERSITY

UNIVERSITY OF NEVADA

NEW MEXICO STATE UNIVERSITY

OREGON STATE UNIVERSITY

UNIVERSITY OF OREGON

OSAKA UNIVERSITY

UNIVERSITY OF SOUTHERN CALIFORNIA
STANFORD UNIVERSITY

UNIVERSITY OF TOKYO

UNIVERSITY OF UTAH

WASHINGTON STATE UNIVERSITY

UNIVERSITY OF WASHINGTON

AMERICAN MATHEMATICAL SOCIETY CALIFORNIA RESEARCH CORPORATION SPACE TECHNOLOGY LABORATORIES NAVAL ORDNANCE TEST STATION 


\section{Pacific Journal of Mathematics}

\section{Vol. 14, No. 1 \\ May, 1964}

Richard Arens, Normal form for a Pfaffian .........................

Charles Vernon Coffman, Non-linear differential equations on cones in Banach

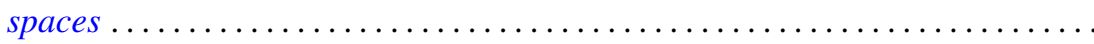

Ralph DeMarr, Order convergence in linear topological spaces ..............

Peter Larkin Duren, On the spectrum of a Toeplitz operator ................

Robert E. Edwards, Endomorphisms of function-spaces which leave stable all

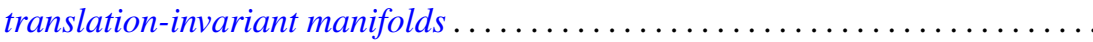

Erik Maurice Ellentuck, Infinite products of isols . . . . . . . . . . . . . . . . 49

William James Firey, Some applications of means of convex bodies . . . . . . . . 53

Haim Gaifman, Concerning measures on Boolean algebras ............. 61

Richard Carl Gilbert, Extremal spectral functions of a symmetric operator. . . . . . 75

Ronald Lewis Graham, On finite sums of reciprocals of distinct nth powers ..... 85

Hwa Suk Hahn, On the relative growth of differences of partition functions ...... 93

Isidore Isaac Hirschman, Jr., Extreme eigen values of Toeplitz forms associated

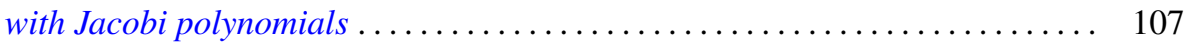

Chen-jung Hsu, Remarks on certain almost product spaces . . . . . . . . . . . 163

George Seth Innis, Jr., Some reproducing kernels for the unit disk . . . . . . . . . 177

Ronald Jacobowitz, Multiplicativity of the local Hilbert symbol . . . . . . . . . . . 187

Paul Joseph Kelly, On some mappings related to graphs ................. 191

William A. Kirk, On curvature of a metric space at a point . . . . . . . . . . . . 195

G. J. Kurowski, On the convergence of semi-discrete analytic functions . . . . . . . 199

Richard George Laatsch, Extensions of subadditive functions . . . . . . . . . . . 209

V. Marić, On some properties of solutions of $\Delta \psi+A\left(r^{2}\right) X \nabla \psi+C\left(r^{2}\right) \psi=0 \ldots 217$

William H. Mills, Polynomials with minimal value sets . . . . . . . . . . . 225

George James Minty, Jr., On the monotonicity of the gradient of a convex



George James Minty, Jr., On the solvability of nonlinear functional equations of 'monotonic' type ................................... 249

J. B. Muskat, On the solvability of $x^{e} \equiv e(\bmod p) \ldots \ldots \ldots \ldots \ldots \ldots \ldots \ldots . \ldots \ldots$

Zeev Nehari, On an inequality of $P . R$. Bessack ................... 261

Raymond Moos Redheffer and Ernst Gabor Straus, Degenerate elliptic

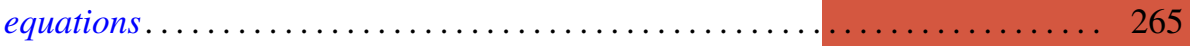

Abraham Robinson, On generalized limits and linear functionals . . . . . . . . . 269

Bernard W. Roos, On a class of singular second order differential equations with a

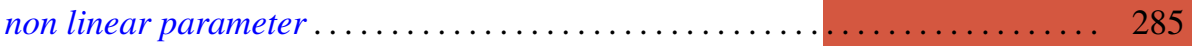

Tôru Saitô, Ordered completely regular semigroups . . . . . . . . . . . . . . . . 295

Edward Silverman, A problem of least area ....................... 309

Robert C. Sine, Spectral decomposition of a class of operators . . . . . . . . . 333

Jonathan Dean Swift, Chains and graphs of Ostrom planes . . . . . . . . . . . 353

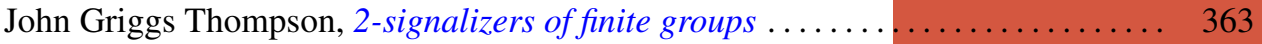

Harold Widom, On the spectrum of a Toeplitz operator . . . . . . . . . . . . . 365 